\title{
Maximal oil recovery by simultaneous condensation of alkane and steam
}

\author{
J. Bruining* \\ Dietz Laboratory, Centre of Technical Geoscience, Mijnbouwstraat 120, 2628 RX Delft, The Netherlands
}

D. Marchesin ${ }^{\dagger}$

Instituto Nacional de Matemática Pura e Aplicada, Estrada Dona Castorina 110, 22460-320 Rio de Janeiro, Rio de Janeiro, Brazil

(Received 4 November 2005; revised manuscript received 12 October 2006; published 26 March 2007)

\begin{abstract}
This paper deals with the application of steam to enhance the recovery from petroleum reservoirs. We formulate a mathematical and numerical model that simulates coinjection of volatile oil with steam into a porous rock in a one-dimensional setting. We utilize the mathematical theory of conservation laws to validate the numerical simulations. This combined numerical and analytical approach reveals the detailed mechanism for thermal displacement of oil mixtures discovered in laboratory experiments. We study the structure of the solution, determined by the speeds and amplitudes of the several nonlinear waves involved. Thus we show that the oil recovery depends critically on whether the boiling-point of the volatile oil is around the water boiling temperature, or much below or above it. These boiling-point ranges correspond to three types of wave structures. When the boiling point of the volatile oil is near the boiling point of water, the striking result is that the speed of the evaporation front is equal or somewhat larger than the speed of the steam condensation front. Thus the volatile oil condenses at the location where the steam condenses too, yielding virtually complete oil recovery. Conversely, if the boiling point is too high or too low, there is incomplete recovery. The condensed volatile oil stays at the steam condensation location because the steam condensation front is a physical shock.
\end{abstract}

DOI: 10.1103/PhysRevE.75.036312

PACS number(s): 44.30.+v, 44.35.+c

\section{INTRODUCTION}

Steam drive is an economical way of producing oil and is used worldwide for heavy oil. An overview of the last forty years of steam drive recovery in California is given in Ref. [1]. Steam drive is also considered an efficient method to clean polluted sites [2-4]. During the steam drive, however, a certain amount of oil is left behind in the steam swept zone [5].

In the late 1970s Dietz [5] proposed to add small amounts of volatile oil to the steam to reduce the oil left behind. Similar ideas were put forward independently by Farouq-Ali [6]. The volatile oil coinjected with the steam in almost infinitesimal amounts would ideally condense at the same location where the steam condenses. The condensed volatile oil acts as a solvent for the heavy oil. As such it pushes the oil away from the steam-swept zone leaving no oil behind (see Fig. 1). At the time the crucial importance of the boiling temperature of the volatile oil was not suspected. Experiments investigating the mechanism are described in Refs. [5-9]. Still, there was a discrepancy between the original idea and the experimental observations. At least $5 \mathrm{wt} \%$ (volatile oil/water) was required to reduce considerably the saturation of the oil left behind [5]. However, it is possible that the requirement of this large percentage was caused by transient effects in the experiments. One of the goals of our work is to clarify this point.

In his pioneering experiment, Willman, in 1961, used a large percentage of initially present volatile oil [9]. His experiment led to the belief that any volatile oil component,

\footnotetext{
*Electronic address: J.bruining@ @itg.tudelft.nl

†Electronic address: marchesi@impa.br
}

initially present in the oil, would lead to virtually complete recovery from the steam-swept zone. Therefore, the virtue of adding volatile oil was criticized at the time. The second goal of our work is to establish the difference between steamdrive recovery with coinjection of volatile oil and recovery of oil already containing a fraction of volatile oil. It can be expected that an efficient condensed volatile oil region is too short for the resolution of standard simulators.

Our approach [10-13] is to simplify the model equations in such a way that the essential elements are retained [14] while avoiding the complexities of solving pressure equations and nonlinear compositional equations at every grid cell. As such the model is a straightforward extension of a one-dimensional (1D) model proposed by Ref. [15], but allowing for immiscible three-phase flow in the steam zone $[16,17]$ (see also Ref. [18]). The simplification is accomplished by the assumption that the steam drive runs at constant pressure as to the thermodynamical behavior; any pressure increase causes an immediate production of fluids. Therefore the pressure equation decouples and we can solve the transport equations locally, reaching resolutions that are unattainable in standard simulators.

The solution of these simplified transport equations is obtained by following each physical state in space time, using the method of characteristics. If the transport equation were linear with constant coefficients all states would move at the same characteristic speed and the wave profiles would remain unchanged. In our case, however, the equations are nonlinear, therefore characteristic speeds depend on the state. If characteristic speeds increase in the flow direction, states spread out giving rise to a rarefaction fan (rarefaction wave). On the other hand, if characteristic speeds decrease the states collapse on each other giving rise to a discontinuity or a shock wave. It is this nonlinear collapse that both generates and stabilizes shock waves. The mathematical theory of 


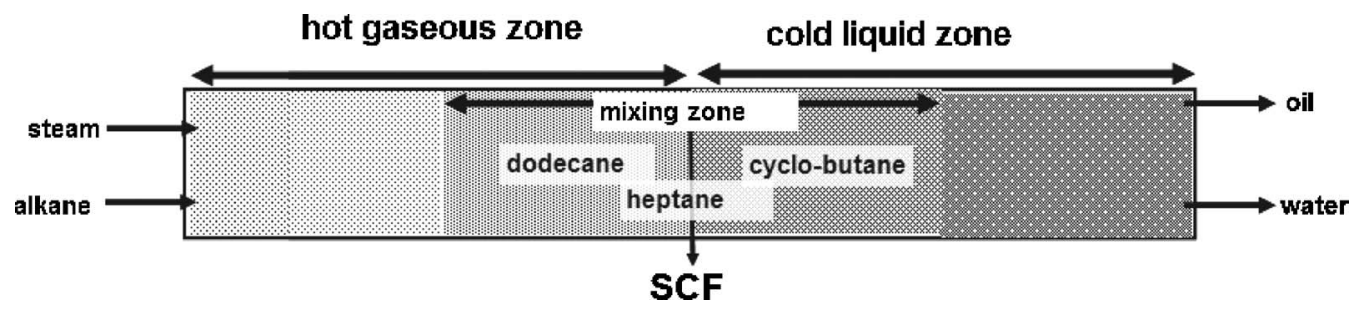

FIG. 1. The porous rock cylinder. Steam and alkane are injected from the left, fluids are produced at the right. In most cases, the hot gaseous zone is sharply separated from the cold liquid zone by the steam condensation front (SCF). Initially the rock is filled with a volatile-oil, dead-oil mixture and water. One of three alkanes is used; dodecane has a tendency to stay upstream of the SCF, and cyclobutane downstream of the SCF; heptane is in between.

these nonlinear waves is very well developed $[19,20]$. However, in our problem mass transfer can occur, viz., evaporation or condensation, giving rise to evaporation or condensation rarefactions or shocks, which are not so well known. The relative speeds of the waves occurring in the solution are crucial in determining the physical phenomena and the distinctive behavior in terms of the values of the physical properties.

Knowing the solution obtained by the method of characteristics has three advantages. Firstly, it is a time-asymptotic solution, which is relevant at the field scale. Secondly, it allows us to validate the numerical solution. Thirdly, it allows the study of bifurcation phenomena, i.e., change of structure of solutions under different injection conditions. The bifurcations of this model in the absence of thermal effects are described in Refs. [21-24]. (See also the review in the appendix of Ref. [25]).

The model we used carries three important simplifications. Firstly, the diffusion mixing between volatile oil and heavy oil in the liquid phase and between volatile oil vapor and water vapor in the gaseous phase are disregarded. The model is not valid for extremely low injection rates, where capillary diffusion dominates convection, because we ignore capillary effects. Finally, we do not specify a detailed model for the kinetics of the condensation process [13]. These aspects determine the internal structure of the shocks, which sometimes affect the structure of the whole Riemann solution, and are subjects for future work [23].

The range of validity of these simplifications can be expressed in terms of dimensionless numbers [26]. For diffusion effects to be negligible, both Péclet numbers [Pe $\left.=\operatorname{Lu}^{i n j} /\left(\varphi D_{i}\right)\right]$, i.e., the one based on molecular diffusion $\left(D_{1}\right)$ and the one based on capillary diffusion $\left(D_{2}\right)$, must be much larger than one. For field conditions $L$ is the distance between wells. The Péclet number is at least one million, even for capillary-diffusion phenomena. For laboratory conditions it is a factor 100 smaller, but still $\mathrm{Pe} \gg 1$. Thirdly, the ratio between the rate of mass transfer between phases and convective mass transport, expressed by the Damkohler number [26], must be very big so that the thickness of the condensation zone can be disregarded. This aspect is discussed in Ref. [27], where it is shown that a practical value of the Damkohler number $\mathrm{Da}=q_{b} L / u^{i n j}$ would be of the order of $10^{8}$. Here $q_{b}\left[s^{-1}\right]$ is the rate of steam condensation. In the same paper it is shown that local equilibrium is obtained when $\mathrm{Da} \sim 10^{4}$. So the condition of local thermodynamic equilibrium is definitely satisfied at $\mathrm{Da}=10^{8}$. This shows that to leading order our model, where we use local thermodynamic equilibrium and disregard diffusion effects, is correct. However, for more precise and quantitative statements these effects must be analyzed. This is however, beyond the scope of this paper.

Finally we also use Darcy's law without inertia correction, which requires that the Reynolds number $\rho v d_{p} / \mu$ based on the grain size, is not larger than one. A typical value for field conditions is $\operatorname{Re}=0.007$. Therefore for field situations these conditions are always satisfied and they were satisfied in most of our laboratory experiments.

Section II describes the physical model and the relevant thermodynamical relations. The flow is described by balance equations in Sec. III. Self-similar waves, i.e., rarefaction and shocks, are analyzed in Sec. IV. An implicit finite difference method requiring the solution of small matrices is described in Sec. V. Section VI summarizes earlier results on the injection of steam displacing heavy oil. Our results concerning the solution structure and the recovery in terms of the boiling point of volatile oil are described and discussed in Sec. VII. We summarize our conclusions in Sec. VIII. Appendix A contains further details. Appendix B describes physical quantities, symbols, and values. Some calculations are found in Appendixes C and D.

\section{PHYSICAL MODEL}

\section{A. Flow of fluids}

The model is based on conventional models for steam drive $[28,29]$. We consider the injection of steam and volatile oil into a linear horizontal porous rock cylinder with constant porosity and absolute permeability (see Fig. 1). The tube is completely thermally isolated. The injection temperature is determined by the three-phase equilibrium condition for the given volatile-oil and steam injection ratio. The cylinder is originally filled with oil and water. The oil consists of dead oil, i.e., oil with a negligible vapor pressure, possibly with dissolved volatile oil. The dissolution of volatile or dead oil in water is negligible. Three-phase flow occurs in the high temperature zone, while oil and water flow occurs in the low temperature zone. The fluids are in local thermodynamic equilibrium. Physical quantities are evaluated at a representative constant pressure throughout the cylinder; this is a good approximation if the total pressure variation is small relative to the total pressure. It is certainly valid in laboratory 
experiments. Thermal expansion of liquids is disregarded. The liquid volatile oil and dead oil heat capacities are not the experimental heat capacities, but slightly adapted so that the enthalpy of the oleic phase is independent of composition. This minor adjustment leads to a major simplification of the mathematical analysis. All fluids are considered incompressible. We assume Darcy's law for multiphase flow [30,31]. The cylinder diameter is sufficiently small so that capillary forces equalize the saturation in the transverse radial direction and temperature is homogeneous radially. As the flow is horizontal we ignore gravity effects.

\section{B. Thermodynamic fundamentals}

Our interest is confined to (1) three-phase flow, i.e., flow of the aqueous $(w)$, oleic $(o)$, and gaseous $(g)$ phases in the steam zone and (2) two-phase flow, i.e., flow of the aqueous and oleic phases in the liquid zone. For liquids, we distinguish between an aqueous (waterlike) phase and an oleic (oil-like) phase because they do not mix. We use the following convention: the first subscript $(w, o, g)$ refers to the phase, the second subscript $(w, v, d)$ refers to the component, i.e., water, volatile oil, and dead oil. Capital subscripts $(W, V, D)$ are used to denote phases consisting of a single component. The densities of the pure liquids are denoted as $\rho_{W}, \rho_{V}$, and $\rho_{D}$. The densities of the pure vapors, i.e., water and volatile oil are denoted by $\rho_{g W}, \rho_{g V}$.

We disregard any heat or volume contraction effects resulting from mixing. The concentration $\left[\mathrm{kg} / \mathrm{m}^{3}\right]$ of volatile (dead) oil in the oleic phase is denoted as $\rho_{o v}\left(\rho_{o d}\right)$. The concentration of water vapor (volatile oil) in the gaseous phase is $\rho_{g w}\left(\rho_{g v}\right)$. For ideal fluids we obtain

$$
\frac{\rho_{o v}}{\rho_{V}}+\frac{\rho_{o d}}{\rho_{D}}=1, \quad \frac{\rho_{g w}}{\rho_{g W}}+\frac{\rho_{g v}}{\rho_{g V}}=1 .
$$

The densities of the pure liquids $\rho_{V}, \rho_{D}\left[\mathrm{~kg} / \mathrm{m}^{3}\right]$ are considered to be independent of temperature, and the densities of the pure vapors to obey the ideal gas law, i.e.,

$$
\rho_{g W}=\frac{M_{W} P}{R T}, \quad \rho_{g V}=\frac{M_{V} P}{R T},
$$

where $M_{W}, M_{V}$ denote the molar weights of water and volatile oil, respectively. $T$ is the temperature and the gas constant is $R=8.31[\mathrm{~J} / \mathrm{mol} \mathrm{K}] . P$ is not a variable in this problem, but the fixed prevailing pressure value; here we use one atmosphere, because most of the experiments were carried out at atmospheric pressure.

The water vapor pressure $P_{w}$ is determined by the Clausius-Clapeyron equation [32]

$$
P_{w}(T)=P_{o} \exp \left[\frac{-M_{W}}{R} \Lambda_{W}\left(T_{b}^{w}\right)\left(\frac{1}{T}-\frac{1}{T_{b}^{w}}\right)\right],
$$

where $\Lambda_{W}\left(T_{b}^{w}\right)[\mathrm{J} / \mathrm{kg}]$ is its evaporation heat at its normal boiling temperature $T_{b}^{w}(\mathrm{~K})$ at $P_{o}$, the atmospheric pressure. We also use Clausius-Clapeyron for the volatile-oil vapor pressure. In addition, we use Raoult's law [32], which states that the vapor pressure of volatile oil is equal to its pure vapor pressure times the mole fraction $x_{o v}$ of volatile oil in the oil phase. Therefore we obtain

$$
P_{v}(T)=x_{o v} P_{o} \exp \left[\frac{-M_{V}}{R} \Lambda_{V}\left(T_{b}^{v}\right)\left(\frac{1}{T}-\frac{1}{T_{b}^{v}}\right)\right],
$$

where $\Lambda_{V}\left(T_{b}^{v}\right)$ is the evaporation heat of the volatile oil at its normal boiling temperature $T_{b}^{v}$. We assume that the prevailing pressure $P$ is the sum of the two vapor pressures. From Eqs. (3) and (4), and $P=P_{w}(T)+P_{v}(T)$, we find for the mole fraction of volatile oil in the liquid-oil phase $x_{o v}(T)$ $=\left(\rho_{o v} / M_{V}\right) /\left(\rho_{o v} / M_{V}+\rho_{o d} / M_{D}\right)$,

$$
x_{o v}(T)=\frac{P-P_{o} \exp \left[\frac{-M_{W}}{R} \Lambda_{W}\left(T_{b}^{w}\right)\left(\frac{1}{T}-\frac{1}{T_{b}^{w}}\right)\right]}{P_{o} \exp \left[\frac{-M_{V}}{R} \Lambda_{V}\left(T_{b}^{v}\right)\left(\frac{1}{T}-\frac{1}{T_{b}^{v}}\right)\right]} .
$$

From this we derive an expression for the volatile-oil concentration in the oleic phase,

$$
\rho_{o v}=\frac{x_{o v} \rho_{D} \rho_{V} M_{V}}{x_{o v} \rho_{D} M_{V}+\left(1-x_{o v}\right) \rho_{V} M_{D}} .
$$

Note that in the gaseous phase no dead oil component is present, whereas in the oleic phase volatile and dead oil are present. Figure 2 shows the projections of the phase diagram of cyclobutane (left), and heptane (right) on the plane of the temperature vs the volatile-oil mole fraction. The special three-phase point " $3 \mathrm{ph}$ " indicates where pure liquid volatile oil, liquid water, and vapor coexist. Other three-phase points are on $\Gamma$, the curve where liquid water, volatile-oil and deadoil mixtures, and vapor coexist as explained below. For each $T$, the mole fraction of volatile oil in the vapor phase on $\Gamma$ is indicated by $y_{g v}=\left[P-P_{w}(T)\right] / P$. This equation is used for heptane in Fig. 2 (right) to find the lower branch $\Gamma$ extending from $\left(y_{g v}, T\right)=[0.0,373.15(\mathrm{~K})] \rightarrow(0.551,351.71)$. At the latter point $x_{o v}=1$. Similarly, the lower branch $\Gamma$ extends from $\left(y_{g v}, T\right)=[0.0,373.15(\mathrm{~K})] \rightarrow(0.983,285.20)$ for cyclobutane [Fig. 2 (left)]. For dodecane it extends from $\left(y_{g v}, T\right)=[0.0,373.15(\mathrm{~K})] \rightarrow(0.0356,371.98)$. All its features of interest occur near the very small branch at the left, which makes a figure less illustrative. Therefore we do not show it. In Fig. 2 we assume that the prevailing pressure is atmospheric, i.e., $P=P_{o}$.

Furthermore, Fig. 2 contains projections of 3D figures with the temperature as the vertical axis, the volatile-oil fraction in the vapor phase, $y_{g v}$ as the horizontal axis, and the composition of the oil phase $x_{o v}$ as the axis perpendicular to the paper. The projection is made on a surface for which $x_{o v}=$ constant.

Figure 2 (left) contains four phase diagrams for $x_{o v}$ $=1,0.6,0.2,0.1$. Consider as an example the phase diagram for $x_{o v}=0.2$, i.e., the behavior of liquid oil with a volatile-oil mole fraction of $x_{o v}=0.2$. This phase diagram consists of the curve $\left\{x_{o v}=0.2\right\}$ (see the next paragraph) and the part of the curve $\Gamma$ to the left of their intersection point. Below these curves the system consists of two liquid phases, where the oleic phase with $x_{o v}=0.2$ is in equilibrium with water. At the 

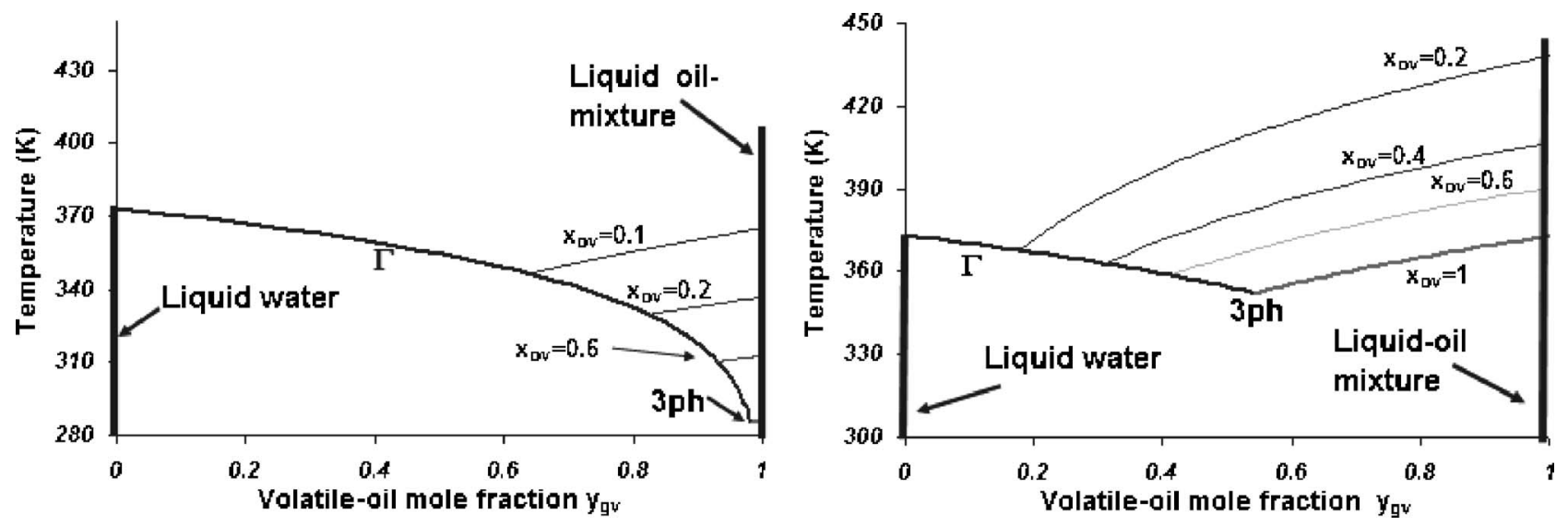

FIG. 2. (Left) Phase diagram for water, dead oil, and cyclobutane. (Right) Phase diagram for water, dead oil, and heptane. The three-phase point $(3 \mathrm{ph})$ for the water-pure volatile-oil system is indicated.

intersection point of these two curves, liquid water and liquid-oil phase with composition $x_{o v}=0.2$ are in equilibrium with vapor with a volatile-oil fraction $y_{g v}$ indicated in the horizontal axis. On $\Gamma$, left of the intersection point liquid water is in equilibrium with vapor with a volatile-oil fraction $y_{g v}$ read from the horizontal axis. Above these curves there is only vapor. Because in all cases considered here there is liquid water, we only use the left curve, the curve $\Gamma$, for the three-phase zone and the region underneath for the twophase zone.

The procedure to find the branches on the right emanating from $\Gamma$ for which $x_{o v}=0.2,0.4,0.6,1.0$ is the following. We pick a value for $x_{o v}$, choosing a curve among these branches. This curve is described by the graph of $y_{g v}(T)=P_{v}(T) / P$, where $P_{v}(T)$ is obtained from Eq. (4). Recall that we used $y_{g v}(T)=\left[P-P_{w}(T)\right] / P$ to obtain the plot of $\Gamma$. Only at the intersection point of the curves liquid oil with the chosen $x_{o v}$, liquid water and vapor are in equilibrium and we have that $y_{g v}(T)=\left[P-P_{w}(T)\right] / P=P_{v}(T) / P$. On $\left\{x_{o v}=1\right\}$ there is no dead oil, rather there is volatile-oil vapor besides water vapor and liquid volatile oil. For $x_{o v}<1$ all branches to the right of $\Gamma$ contain dead oil too. Therefore, these branches with constant $x_{o v}$ describe the two-phase oleic-gaseous equilibrium; there is no liquid water. We can use Eq. (5) to obtain expressions for the concentrations $\rho_{o v}(T), \rho_{o d}(T), \rho_{g w}(T)$, and $\rho_{g v}(T)$.

\section{BALANCE EQUATIONS}

The energy conservation equation in terms of enthalpy is given as [33]

$$
\begin{aligned}
\frac{\partial}{\partial t}\left[H_{r}\right. & \left.+\varphi\left(H_{W} S_{w}+H_{o} S_{o}+H_{g} S_{g}\right)\right] \\
& +\frac{\partial}{\partial x} u\left(H_{W} f_{w}+H_{o} f_{o}+H_{g} f_{g}\right)=0,
\end{aligned}
$$

where the enthalpies per unit volume $H_{W}, H_{o}$, and $H_{g}$ are defined in the table in terms of densities and enthalpies per unit mass $h_{W}, h_{o V}, h_{o D}, h_{g V}, h_{g W}$. These enthalpies depend on temperature (and on the fixed pressure). The enthalpy of steam in the gaseous phase is $h_{g W}$, and $h_{W}$ is the enthalpy of water in the aqueous phase, while $h_{g V}$ is the enthalpy of volatile oil in the gaseous phase. Furthermore, $h_{o V}$ and $h_{o D}$ are the enthalpies of liquid volatile oil and dead oil. Their values are chosen so that the heat capacity per unit volume $H_{o}$ is only a function of temperature (see Appendix B). The rock enthalpy $H_{r}$ is per unit volume. The saturation of the oleic, aqueous, and gaseous phases are $S_{o}, S_{w}, S_{g}$, while $f_{o}$, $f_{w}, f_{g}$ are their fluxes, defined in Eq. (B16). We use $u$ to denote the total Darcy flow velocity and $\varphi$ the constant rock porosity. We can write for the mass conservation equations of water, volatile oil, and total oil [34],

$$
\begin{gathered}
\varphi \frac{\partial}{\partial t}\left(\rho_{g w} S_{g}+\rho_{W} S_{w}\right)+\frac{\partial}{\partial x} u\left(\rho_{g w} f_{g}+\rho_{W} f_{w}\right)=0, \\
\varphi \frac{\partial}{\partial t}\left(\rho_{g v} S_{g}+\rho_{o v} S_{o}\right)+\frac{\partial}{\partial x} u\left(\rho_{g v} f_{g}+\rho_{o v} f_{o}\right)=0, \\
\varphi \frac{\partial}{\partial t}\left(\frac{\rho_{g v}}{\rho_{V}} S_{g}+S_{o}\right)+\frac{\partial}{\partial x} u\left(\frac{\rho_{g v}}{\rho_{V}} f_{g}+f_{o}\right)=0 .
\end{gathered}
$$

Equations (8) and (7) can be written in condensed form as

$$
\frac{\partial}{\partial t} G_{\ell}+\frac{\partial}{\partial x} u F_{\ell}=0 \quad \text { for } \ell=w, v, o, T .
$$

We use the subscript $\ell$ to denote the components $(w, v, o)$ and the energy $(T)$. Notice that in the three-phase zone, $G_{\ell}$ and $F_{\ell}$ are functions of the variables $S_{w}, S_{g}, T$ and the dependent variables of Eq. (9) are $S_{w}, S_{g}, T$, and $u$. In the two-phase zone Eqs. (8) and (7) simplify by using $\rho_{g w}=\rho_{g v}$ $=0$. Here $f_{w}$ depends on $S_{w}$ and $T, f_{o}$ on $S_{w}, \rho_{o v}$, and $T$. The dependent variables in the two-phase zone in Eq. (9) are $S_{w}$, $\rho_{o v}, T$, and $u$. Thus a state in the three-phase zone is defined by the values of $S_{w}, S_{g}, T, u$ and in the two-phase zone by the values of $S_{w}, \rho_{o v}, T, u$.

\section{ANALYSIS OF ELEMENTARY WAVES}

Considering Eq. (9) and the fact that we use constant injection conditions and homogeneous initial data we ob- 
serve that solutions must exist that are invariant with respect to scaling $x \rightarrow a x, t \rightarrow a t$, where $a$ is any positive constant. Such solutions depend only on the similarity coordinate $x / t$ and are called Riemann solutions. These solutions represent large-time asymptotic solutions for many initial and boundary data. Standard theory of conservation laws say that Riemann solutions consist of sequences of smooth rarefaction waves, discontinuities or shocks, and constant states. Shock waves satisfy the Rankine-Hugoniot (RH) conditions, which express mass conservation. We refer the interested reader to Smoller [20] and Dafermos [19]. Excellent engineering introductions in this field can be found in the papers by Pope [35], Hirasaki [36], and Dumoré, Hagoort, and Risseeuw [37], and in the book by Lake [34].

The theory of nonlinear conservation laws relates the speed of a shock with its left and right states through the RH conditions. We find explicit formulas for RH conditions for all shocks, including condensation shocks, one of which is the steam condensation front (SCF). We derive the characteristic speeds for rarefaction waves. We have also obtained the rarefaction curves, which represent the rarefaction waves, but we omit their lengthy derivations here. We have used these formulas to verify the correctness of every single wave found numerically in Sec. VII. The concatenation of the waves according to speed and the extended Lax entropy conditions $[20,19]$ were verified as well. As far as the authors are concerned the treatment of the velocity variable is original as there are no time derivatives for this variable in the equations.

\section{A. Shocks}

We use the standard notation for the jump of a quantity $U$ across a shock as $[U]=U^{+}-U^{-}$. The RH conditions for Eqs. (8) and (7) can be written as follows [for a shock with speed $v$ and left and right states $(-)$ and $(+)]$ :

$$
\begin{gathered}
-\varphi v\left[\rho_{g w} S_{g}+\rho_{W} S_{w}\right]+\left[u\left(\rho_{g w} f_{g}+\rho_{W} f_{w}\right)\right]=0, \\
-\varphi v\left[\rho_{g v} S_{g}+\rho_{o v} S_{o}\right]+\left[u\left(\rho_{g v} f_{g}+\rho_{o v} f_{o}\right)\right]=0, \\
-\varphi v\left[\frac{\rho_{g v}}{\rho_{V}} S_{g}+S_{o}\right]+\left[u\left(\frac{\rho_{g v}}{\rho_{V}} f_{g}+f_{o}\right)\right]=0, \\
-v\left[H_{r}+\varphi\left(H_{W} S_{w}+H_{o} S_{o}+H_{g} S_{g}\right)\right] \\
+\left[u\left(H_{W} f_{w}+H_{o} f_{o}+H_{g} f_{g}\right)\right]=0 .
\end{gathered}
$$

When $v, u^{+}, u^{-}$solve the equations above, then $a v, a u^{+}, a u^{-}$ also solve the equations.

We distinguish six kinds of shocks. (1) The volatile-oil evaporation shock (speed $v_{E}$ ), with three-phase conditions at the left. Its main feature is that the volatile-oil concentration increases in the downstream (right) direction. The temperature, saturations, and velocity change across the shock. (2) The steam condensation shock (speed $v_{S C F}$ ), with a threephase condition at the left. The vapor saturation decreases drastically in the downstream direction. Again all the quantities change across the shock. (3) The volatile-oil condensation shock (speed $v_{C}$ ), with a three-phase condition at the left. The volatile-oil concentration decreases in the downstream direction. (4) The volatile-oil two-phase composition shock (speed $v_{v}$ ), which is a contact discontinuity. A contact discontinuity represents the moving interface between two fluids in the same phase. In reality such an interface is not infinitesimally thin. In loose mathematical terms, a contact discontinuity is defined as a shock for which the characteristic speeds at the right and left are equal to the shock speed. (5) The saturation shock (speed $v_{S}$ ). Only the saturations change, while temperature, composition, and the velocity are constant, so that Eq. (13) does not play a role. (6) The Buckley-Leverett shock (speed $v_{B L}$ ), with only the liquid oil and water phases present. All quantities except the liquid saturations are constant, so that Eq. (13) again does not play a role.

\section{B. Characteristic speeds}

Using $G_{\ell}$ and $F_{\ell}$ from Eq. (9), we define

$$
G_{\ell n}=\frac{\partial G_{\ell}}{\partial V_{n}}, \quad F_{\ell n}=\frac{\partial F_{\ell}}{\partial V_{n}}, \quad F_{\ell u}=\frac{\partial}{\partial u}\left(u F_{\ell}\right)=F_{\ell},
$$

where $V=\left(V_{1}, V_{2}, V_{3}\right)=\left(S_{w}, S_{g}, T\right)$. Note that $G_{\ell}$ does not depend on $u$. Without loss of generality $u>0$ and Eq. (9) can be rewritten for $\ell=w, v, o, T$ as

$$
\sum_{n=w, g, T}\left(G_{\ell n} \frac{\partial V_{n}}{\partial t}+u F_{\ell n} \frac{\partial V_{n}}{\partial x}\right)+F_{\ell} u \frac{\partial \ln u}{\partial x}=0 .
$$

Let us consider solutions of Eq. (9) that depend on $(x, t)$ through the similarity coordinate $\eta=x / t$. Then Eq. (15) with $\left(S_{w}, S_{g}, T, \ln u\right)^{\dagger}$ denoting a column vector becomes

$$
M\left(S_{w}, S_{g}, T, \frac{\eta}{u}\right) \frac{d}{d \eta}\left(S_{w}, S_{g}, T, \ln u\right)^{\dagger}=0,
$$

where $M(V, \lambda)$ with $\lambda=\eta / u$ is the $4 \times 4$ matrix

$$
\left(\begin{array}{cccc}
F_{11}-\lambda G_{11} & F_{12}-\lambda G_{12} & F_{13}-\lambda G_{13} & F_{1} \\
F_{21}-\lambda G_{21} & F_{22}-\lambda G_{22} & F_{23}-\lambda G_{23} & F_{2} \\
F_{31}-\lambda G_{31} & F_{32}-\lambda G_{32} & F_{33}-\lambda G_{33} & F_{3} \\
F_{41}-\lambda G_{41} & F_{42}-\lambda G_{42} & F_{43}-\lambda G_{43} & F_{4}
\end{array}\right) \text {. }
$$

We have replaced the subscripts $w, v, o, T$ by $1,2,3,4$. Explicit expressions for the flux functions, the accumulation function, and their derivatives can be found in Appendix D.

\section{Three-phase flow}

We find the characteristic speeds, viz., the eigenvalues $\lambda$ of $M$. Making the determinant of Eq. (17) equal to zero leads to a polynomial equation of third order in $\lambda$. Indeed, after performing Gaussian elimination on the matrix (17), its determinant becomes the following: 


$$
\begin{array}{|}
\left|\begin{array}{cccc}
\frac{\partial f_{w}}{\partial S_{w}}-\lambda \varphi & \frac{\partial f_{w}}{\partial S_{g}} & A_{13}(\lambda) & A_{1} \\
\frac{\partial f_{g}}{\partial S_{w}} & \frac{\partial f_{g}}{\partial S_{g}}-\lambda \varphi & A_{33}(\lambda) & A_{3} \\
0 & 0 & A_{23}^{0}-\lambda A_{23}^{1} & A_{2} \\
0 & 0 & \mathcal{F}_{43}-\lambda \mathcal{G}_{43} & \mathcal{F}_{4}
\end{array}\right| \\
=\left|\begin{array}{cc}
\frac{\partial f_{w}}{\partial S_{w}}-\lambda \varphi & \frac{\partial f_{w}}{\partial S_{g}} \\
\frac{\partial f_{g}}{\partial S_{w}} & \frac{\partial f_{g}}{\partial S_{g}}-\lambda \varphi
\end{array}\right|\left|\begin{array}{cc}
A_{23}^{0}-\lambda A_{23}^{1} & A_{2} \\
\mathcal{F}_{43}-\lambda \mathcal{G}_{43} & \mathcal{F}_{4}
\end{array}\right|,
\end{array}
$$

where $A_{1}, A_{3}, A_{2}, A_{23}^{0}, A_{23}^{1}, \mathcal{F}_{4}, \mathcal{F}_{43}$, and $\mathcal{G}_{43}$ depend on $V$, while $A_{13}(\lambda), A_{33}(\lambda)$ are linear expressions in $\lambda$ with coefficients that depend on $V$. The calculation and expressions are found in Appendix $\mathrm{C}$.

Let us define $D=\left(\frac{\partial f_{w}}{\partial S_{w}}-\frac{\partial f_{g}}{\partial S_{g}}\right)^{2}+4 \frac{\partial f_{w}}{\partial S_{g}} \frac{\partial f_{g}}{\partial S_{w}}$ and $T=\frac{\partial f_{w}}{\partial S_{w}}+\frac{\partial f_{g}}{\partial S_{g}}$. The slow and fast characteristic speeds for saturation rarefaction waves are given as

$$
\lambda_{S, 1}=\frac{-T-\sqrt{D}}{2 \varphi}, \quad \lambda_{S, 2}=\frac{-T+\sqrt{D}}{2 \varphi} .
$$

The corresponding characteristic vectors have constant $T, u$; only the saturations vary along these waves. Within the saturation triangle, spanned by $S_{w}, S_{o}, S_{g}$, which add to one, there is a point where $X_{1}=0$. Here the two characteristic speeds coincide, giving rise to a rich wave structure (see, e.g., Ref. [24]).

The third and last characteristic speed is

$$
\lambda_{c}=\lambda_{c}\left(S_{w}, S_{g}, T\right)=\frac{1}{\varphi} \frac{A_{2} \mathcal{F}_{43}-A_{23}^{0} \mathcal{F}_{4}}{A_{2} \mathcal{G}_{43}+A_{23}^{1} \mathcal{F}_{4}} .
$$

This is associated with a condensation rarefaction wave, in which all quantities vary.

\section{Two-phase flow}

In the absence of the gaseous phase, there are three kinds of rarefaction waves. One is the thermal rarefaction wave, along which $S_{w}, \rho_{o v}, T$ change. Its speed is

$$
\lambda_{T}=\frac{H_{o}^{\prime}+\left(H_{w}^{\prime}-H_{o}^{\prime}\right) f_{w}}{H_{r}^{\prime}+\varphi\left[H_{o}^{\prime}+\left(H_{w}^{\prime}-H_{o}^{\prime}\right) S_{w}\right]} .
$$

Then we have the Buckley-Leverett rarefaction with speed $\lambda_{B L}$, along which the liquid saturation $S_{w}$ changes. Finally, we have the composition wave with speed $\lambda_{C}$, which is a contact discontinuity, along which the composition and the liquid-water saturation change. The speeds $\lambda_{B L}\left(S_{w}, \rho_{o v}, T\right)$ and $\lambda_{C}\left(S_{w}, \rho_{o v}, T\right)$ are

$$
\lambda_{B L}=\frac{1}{\varphi} \frac{\partial f_{w}}{\partial S_{w}}, \quad \lambda_{C}=\frac{1}{\varphi} \frac{1-f_{w}}{1-S_{w}} .
$$

We have computed the characteristic vectors for both twoand three-phase flow, but we do not provide the formulas here. They are necessary to compute the rarefaction waves.

\section{NUMERICAL SOLUTION OF THE EQUATIONS}

We will use the notation $V=\left(S_{w}, S_{g}, T\right)$ in the three-phase region and $V=\left(S_{w}, v_{o v}, T\right)$ in the two-phase liquid region.

\section{A. Upstream scheme}

Consider Eq. (9), where the fluxes $F_{\ell}$ are functions of $V$. We can write the upstream implicit finite-difference scheme $(\ell=w, v, o, T)$

$$
\begin{aligned}
& G_{\ell}^{m}(t+\Delta t)+u^{m}(t+\Delta t) F_{\ell}^{m}(t+\Delta t) \\
& \quad=G_{\ell}^{m}(t)+(\Delta t / \Delta x) u^{m-1}(t+\Delta t) F_{\ell}^{m-1}(t+\Delta t),
\end{aligned}
$$

where $m$ denotes the grid-cell number. The unknowns are $u^{m}(t+\Delta t)$ and the three components of $V^{m}(t+\Delta t)$, which show up in the expressions for $G_{\ell}^{m}(t+\Delta t)$ and $F_{\ell}^{m}(t+\Delta t)$.

Let us rewrite Eq. (23) and shorten the unknowns as follows: $u^{m}(t+\Delta t)$ as $u$ and $V^{m}(t+\Delta t)$ as $\mathcal{V}$. We obtain the nonlinear implicit scheme

$$
G_{\ell}(\mathcal{V})+(\Delta t / \Delta x) u F_{\ell}(\mathcal{V})=R_{\ell}^{m, m-1},
$$

where we have introduced the notation $R_{\ell}^{m, m-1}$ for the righthand side of Eq. (23). We assume that $F_{\ell}^{m-1}(t+\Delta t)$ and $u^{m-1}(t+\Delta t)$ have been precomputed when solving the previous cell $m-1$, which may be in a phase condition different from that of cell $m$. We emphasize that $R_{\ell}^{m, m-1}$ does not depend on the condition of cell $m$ at the new time $t+\Delta t$.

\section{B. Solution of the nonlinear system}

The system (24) is solved using Newton-Raphson. Given an approximate solution in the $k$ th iteration $\mathcal{V}^{k}$ and $u^{k}$ of Eq. (24), we find a better approximation in the $(k+1)$ th iteration. Equation (24) becomes

$$
0=G_{\ell}\left(\mathcal{V}^{k+1}\right)+(\Delta t / \Delta x) u^{k+1} F_{\ell}\left(\mathcal{V}^{k+1}\right)-R_{\ell}^{m, m-1} .
$$

Substituting $\mathcal{V}^{k+1}=\mathcal{V}^{k}+d \mathcal{V}, u^{k+1}=u^{k}+d u$, and neglecting second-order terms we obtain

$$
\left(\frac{\partial G_{\ell}}{\partial \mathcal{V}}\left(\mathcal{V}^{k}\right)+\frac{\Delta t}{\Delta x} u^{k} \frac{\partial F_{\ell}}{\partial \mathcal{V}}\left(\mathcal{V}^{k}\right)\right) d \mathcal{V}+\frac{\Delta t}{\Delta x} F_{\ell}\left(\mathcal{V}^{k}\right) d u=-\mathcal{R}_{\ell}^{k},
$$

where we have defined $R_{\ell}^{k}$ as

$$
\mathcal{R}_{\ell}^{k}=G_{\ell}\left(\mathcal{V}^{k}\right)+(\Delta t / \Delta x) u^{k} F_{\ell}\left(\mathcal{V}^{k}\right)-R_{\ell}^{m, m-1} .
$$

This is solvable for $(d \mathcal{V}, d u)$ if $u \Delta t / \Delta x$ is not a characteristic speed, which can be achieved by taking $\Delta t$ small enough. After division of this equation by $u^{k} \Delta t / \Delta x$, we obtain the following linear system to be solved at each Newton iteration, written in the notation of Eq. (17):

$$
M\left(\mathcal{V},-\frac{\Delta x}{u^{k} \Delta t}\right)\left(\begin{array}{c}
d \mathcal{V}_{1} \\
d \mathcal{V}_{2} \\
d \mathcal{V}_{3} \\
d u / u^{k}
\end{array}\right)=\left(\begin{array}{c}
-\mathcal{R}_{1}^{k} \\
-\mathcal{R}_{2}^{k} \\
-\mathcal{R}_{3}^{k} \\
-\mathcal{R}_{4}^{k}
\end{array}\right) .
$$

\section{Numerical implementation}

The quantities in the grid cells are computed in the injection-production direction from the left to the right. We 


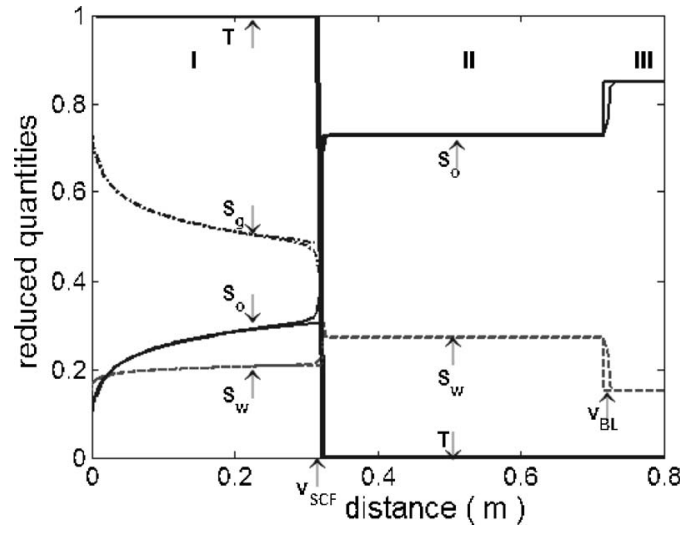

FIG. 3. Comparison of the MOC with the FD solutions for the case that volatile oil is neither injected nor present in the initial oil. The curves with sharp edges are obtained with MOC, and the smoother ones with FD. The saturation curves are indicated as $S_{w}, S_{g}, S_{o}$ in obvious notation. The temperature is indicated with a $T$. Also indicated is the steam condensation front $v_{S C F}$ and the Buckley-Leverett shock $v_{B L}$.

specify the fluxes of all components at the injection boundary. Initially, all cells contain a homogeneous distribution of water and an oleic phase at low temperature.

All calculations in the Newton-Raphson scheme depend on the old phase condition of cell $m$, as well as on available information from cells to the left of cell $m$. The method of solution depends on the new condition of the cell, two-phase or three-phase.

The iterative procedure is simple for a cell that starts and stays in the same condition. When a cell starts in the twophase condition, but in the two-phase calculation a temperature arises that exceeds the boiling temperature of the water and oleic-phase mixture [see Fig. 2 (left)] then the calculation is replaced by a three-phase calculation.

Simulations use a uniform grid with 2000 blocks. This implicit method is inexpensive as it only involves the solution of many $3 \times 3$ matrices as opposed to a single big matrix. As far as the authors are concerned, this upstream box finite difference method is original in the way the total velocity is treated, as there is no time derivative for it in the system. (See Ref. [38] for a related scheme.)

\section{METHOD OF CHARACTERISTICS FOR STEAM INJECTION}

Figure 3 compares the numerical solutions obtained by the current finite-difference scheme (FD) and by the method of characteristics (MOC) used in Ref. [13] for the saturations $\left(S_{w}, S_{o}, S_{g}\right)$ versus the length along the cylinder for pure steam injection in a cylinder filled initially with dead oil only. The profiles are shown after the injection of 0.057 $\mathrm{P}$ (ore) $\mathrm{V}$ (olume) (cold water equivalent). In region I (the steam zone), where $S_{g}>0$, we observe a saturation rarefaction wave, in which the temperature and Darcy velocity are constant. At the steam condensation shock or front (SCF), where the temperature drops to the initial temperature, the gas saturation drops to zero. Here we use the word shock and front interchangeably. The water saturation is larger than the initial water saturation $\left(S_{w c}=0.15\right)$, both in regions I and II. Downstream of region II there is a second shock to the initial conditions, i.e., $S_{w}=S_{w c}, S_{o}=1-S_{w c}$, in region III. The total downstream Darcy velocity divided by the injection velocity is constant spatially at $1.19 \times 10^{-3}$ in the entire liquid zone (regions II and III), but it shows numerical fluctuations of $20 \%$ between time steps. Nevertheless the average is correct. The oil saturation at the SCF is about 0.3. The observed behavior is approximately independent of the number of grid blocks. The reduced temperature is plotted, but the total Darcy velocity is not because they visually coincide.

\section{RESULTS}

We distinguish two classes of results. In the first class the volatile oil is initially present in the reservoir, but it is not coinjected. In the second class no volatile oil is initially present, but it is coinjected with the steam. For each of the classes the volatile oil is cyclobutane, heptane, or dodecane. These alkanes were chosen because they have low, medium, and high boiling points, in such a way that each one gives rise to a different type of solution.

The initial conditions for all the calculations are the following. The initial temperature is $293 \mathrm{~K}$ and the gas saturation is zero. The initial water saturation is given as $S_{w}=S_{w c}$ $=0.15$. We consider the cases where the oleic phase consists of dead oil and of a volumetric 50\% mixture of dead oil and volatile oil; however, for cyclobutane we use a volumetric $20 \%$ mixture of dead oil and volatile oil, as 50\% is above the solubility limit. In the former case we inject an alkane and steam vapor with mass fraction 0.2 [alkane/(alkane + steam)]. In the latter case we displace with pure steam. The injection temperature is $373 \mathrm{~K}$ and the injection pressure is one atmosphere. We use atmospheric pressure because these results are easiest to validate by laboratory experiments. The volumetric injection flux is $9.52 \times 10^{-4} \mathrm{~m} / \mathrm{s}$. From now on, all figures plot reduced quantities versus the distance. The reduced velocity $(u)$ is the total Darcy velocity divided by the injection velocity. The reduced temperature is ( $T$ $\left.-T_{o}\right) /\left(T_{b}^{w}-T_{o}\right)$ (see Appendix B for terminology). The reduced concentration (volume fraction) is $v_{o v}=\rho_{o v} / \rho_{V}$.

Long time runs were subdivided in shorter ones to dampen transient behavior faster; in each one the initial data consisted of the previous one, where every other grid data was omitted [39]. Each run was stopped before breakthrough of the fastest wave, i.e., before it reached the end boundary.

\section{A. Cyclobutane and steam mixture displacing dead oil}

Figure 4 shows displacement of dead oil by steam and cyclobutane. There are four regions from the injection point to the initial condition. In region I, there is a fast three-phase saturation rarefaction wave with speed given by Eq. (19) (right); $T$ and $u$ are constant on this wave; the volatile oil concentration $\left(v_{o v}\right)$ is a small constant. The SCF separates region I from region II. In region II the temperature and the total velocity are constant, but much lower than in region I. Regions I and II are three-phase regions, whereas regions III 


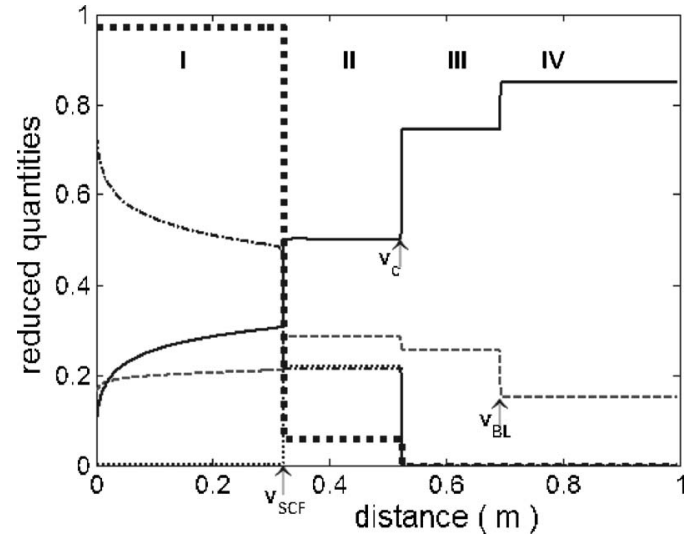

FIG. 4. Steam and cyclobutane displacement of dead oil. The water saturation $S_{w}$, oil saturation $S_{o}$, and steam saturation $S_{g}$ are shown as the dashed, solid, and dashed-dotted curves. The volatileoil fraction $v_{o v}$ in the oil phase and the reduced temperature are shown as the dotted and filled-square curves.

and IV are two-phase liquid regions. Regions II and III are separated by a cyclobutane evaporation shock, where the temperature jumps to its initial value in the reservoir and the total velocity to its final downstream value. There is a constant state in region III. Regions III and IV are separated by a Buckley-Leverett shock. Region IV contains the initial saturations.

The rarefaction in region I starts at the injection state $\left(S_{w c}, 1-S_{w c}, T_{b}^{w}, u^{i n j}\right)$ and ends at the left state of the SCF, $\left(S_{w, \mathrm{SCF}}^{-}, S_{g, \mathrm{SCF}}^{-}, T_{b}^{w}, u^{i n j}\right)$. The right state of the SCF is $\mathrm{SCF}^{+}$ $=\left(S_{w, \mathrm{SCF}}^{+}, S_{g, \mathrm{SCF}}^{+}, T_{\mathrm{SCF}}^{+}, u_{\mathrm{SCF}}^{+}\right)$. Left and right states and $v_{\mathrm{SCF}}$ satisfy the RH conditions (10)-(13). The velocity $v_{\mathrm{SCF}}$ is the same as the speed of fast three-phase rarefaction [Eq. (19)] at the end of region I, i.e., the SCF shock is left-characteristic. Region II starts at $\mathrm{SCF}^{+}$, which is also the upstream (left) state of the cyclobutane condensation shock with speed $v_{C}$. The right state of this shock is $C^{+}=\left(S_{w, C}^{+}, S_{g, C}^{+}=0, T_{o}, u_{C}^{+}\right)$. Left and right states and $v_{C}$ satisfy the RH conditions (10)-(13). Region III is a constant state. Therefore $C^{+}$is the upstream (left) state of the Buckley-Leverett shock. Region IV is a constant state, which is the right state of this shock, with initial reservoir saturation and temperature.

\section{B. Pure steam displacing a dead-oil and cyclobutane mixture}

As shown in Fig. 5, there are four regions again. In region I, there is a fast three-phase saturation rarefaction wave at constant $T$ and $u$. Separating region I from region II, there is the SCF. Note that the temperature at the right side of the $\mathrm{SCF}$ is not the initial reservoir temperature, but an intermediate temperature. Region II consists of a constant state with temperature and total velocity lower than in region I. Regions II and III are separated by a three-phase saturation shock, which does not change the temperature but reduces the water saturation to its initial value. The gas saturation in region III is slightly lower than in region II. Between region III and region IV there is a cyclobutane condensation shock.

Region I $\left(v_{o v}=0\right)$ starts at the injection state $\left(S_{w c}, S_{g}=1\right.$ $\left.-S_{w c}, T_{b}^{w}, u^{i n j}\right)$ and ends at $\left(S_{w, \mathrm{SCF}}^{-}, S_{g, \mathrm{SCF}}^{-}, T_{b}^{w}, u^{i n j}\right)$, which is

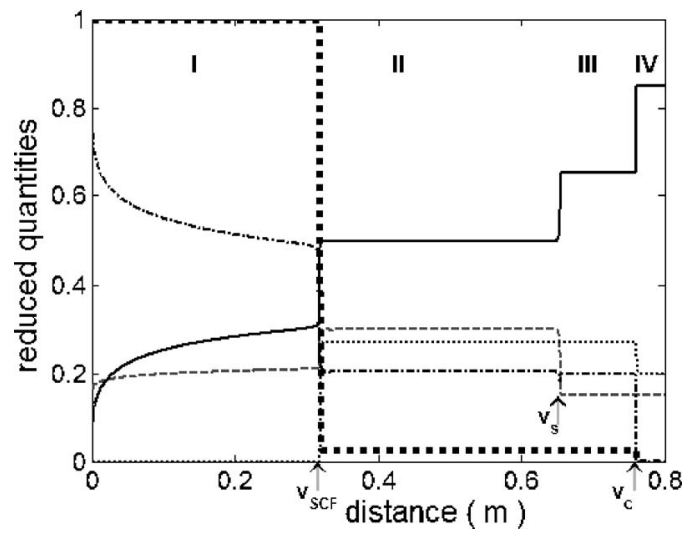

FIG. 5. Steam displacement of a dead-oil or cyclobutane mixture. The water saturation, oil saturation, and steam saturation are shown as the dashed, solid, and dashed-dotted curves. The volatile oil fraction in the oil phase and the reduced temperature are shown as the dotted and filled-square curves.

the left state of the SCF. The right state of the SCF is $\mathrm{SCF}^{+}=\left(S_{w, \mathrm{SCF}}^{+}, S_{g, \mathrm{SCF}}^{+}, T_{\mathrm{SCF}}^{+}, u_{\mathrm{SCF}}^{+}\right)$. Left and right states and $v_{\mathrm{SCF}}$ satisfy the RH conditions (10)-(13). The SCF is left characteristic. Region II consists of the constant state $\mathrm{SCF}^{+}$. Region II ends at the three-phase saturation shock with speed $v_{S}$. The right state is denoted as $\left(S_{w, S}^{+}, S_{g, S}^{+}, T_{\mathrm{SCF}}^{+}, u_{\mathrm{SCF}}^{+}\right)$and continues in region III. This constant state ends at the condensation shock with speed $v_{C}$. Region IV is a constant state, which is the right state of this shock, with initial reservoir saturation and temperature.

\section{Steam and heptane mixture displacing dead oil}

As shown in Fig. 6, in region I there is again a fast threephase saturation rarefaction. At the SCF the temperature drops to the initial temperature, and a volatile-oil bank (region II) builds up downstream of the SCF. The volatile-oil bank does not contain any dead oil. Such a pure volatile-oil bank displaces all dead oil. Downstream of the volatile-oil

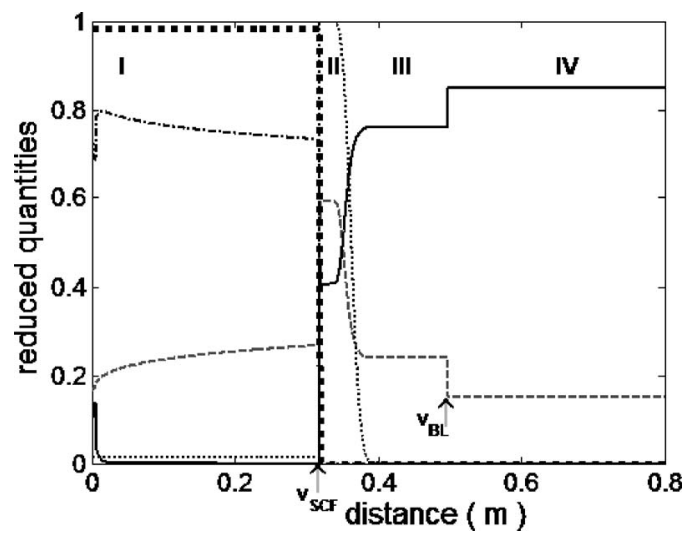

FIG. 6. Steam and heptane displacement of dead oil. The water saturation, oil saturation, and steam saturation are shown as the dashed, solid, and dashed-dotted curves. The volatile oil fraction in the oil phase and the reduced temperature are shown as the dotted and filled-square curves. 


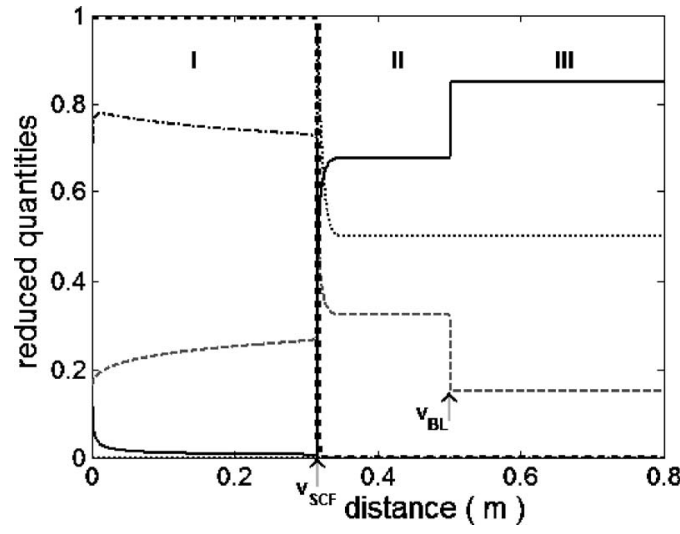

FIG. 7. Steam displacement of a dead-oil and heptane mixture. The water saturation, oil saturation, and steam saturation are shown as the dashed, solid, and dashed-dotted curves. The volatile-oil fraction in the oil phase and the reduced temperature are shown as the dotted and filled-square curves.

bank there is a contact wave, which marks the boundary between regions II and III. The contact wave is smooth in the simulation due to numerical diffusion. Downstream there is only dead oil. Region III consists of a constant state. Regions III and IV are separated by a Buckley-Leverett shock.

Region I starts at the injection state $\left(S_{w c}, S_{g}=1\right.$ $\left.-S_{w c}, T^{i n j}, u^{i n j}\right)$, and it ends at the left state of the SCF, viz., $\left(S_{w, \mathrm{SCF}}^{-}, S_{g, \mathrm{SCF}}^{-}, T^{i n j}, u^{i n j}\right)$. Again the SCF is left characteristic. The right state of the $\mathrm{SCF}$ is $\mathrm{SCF}^{+}=\left(S_{w, \mathrm{SCF}}^{+}, S_{g, \mathrm{SCF}}^{+}, v_{o v}\right.$ $\left.=1, T_{o}, u^{+}\right)$and continues as a constant state in region II. Between regions II and III there is a volatile-oil contact wave with right state $\mathrm{SCF}^{+}$and velocity $v_{v}$. Downstream of the contact wave the constant state $\left(S_{w, C}^{+}, S_{g, C}^{+}, v_{o v}=0, T_{o}, u^{+}\right)$ spans region III. The Buckley-Leverett shock separates region III from region IV. This solution agrees with the observation made previously by Bruining and collaborators in the laboratory experiments $[5,7]$. This is the case when analytical, numerical, and experimental results are all available. They all agree.

\section{Pure steam displacing a dead-oil and heptane mixture}

As shown in Fig. 7, there are only three regions. In region I, there is the usual rarefaction wave with constant $T$ and $u$. Separating region I from region II there is the SCF. In region II the temperature is equal to the initial temperature and the total velocity attains its constant downstream value. At the SCF there is a remarkable spike of volatile oil. The volatile oil concentration $v_{o v}$ vanishes at the left of the SCF, it reaches almost one at the SCF, and then it declines to its initial value. Region II consists of a constant state. Regions II and III are separated by a Buckley-Leverett shock. Region III contains the initial saturations.

Region I starts at the injection state $\left(S_{w c}, S_{g}=1\right.$ $\left.-S_{w c}, T_{b}^{w}, u^{i n j}\right)$ and ends at the left state of the SCF, viz., $\left(S_{w, \mathrm{SCF}}^{-}, S_{g, \mathrm{SCF}}^{-}, T_{b}^{w}, u^{i n j}\right)$. The right state is $\mathrm{SCF}^{+}$ $=\left(S_{w, \mathrm{SCF}}^{+}, S_{g, \mathrm{SCF}}^{+}=0, T_{o}, u^{+}\right)$. Left and right states and $v_{\mathrm{SCF}}$ satisfy the RH conditions (10)-(13). Again the SCF is left characteristic. Region II consists of the constant state $\mathrm{SCF}^{+}$. A

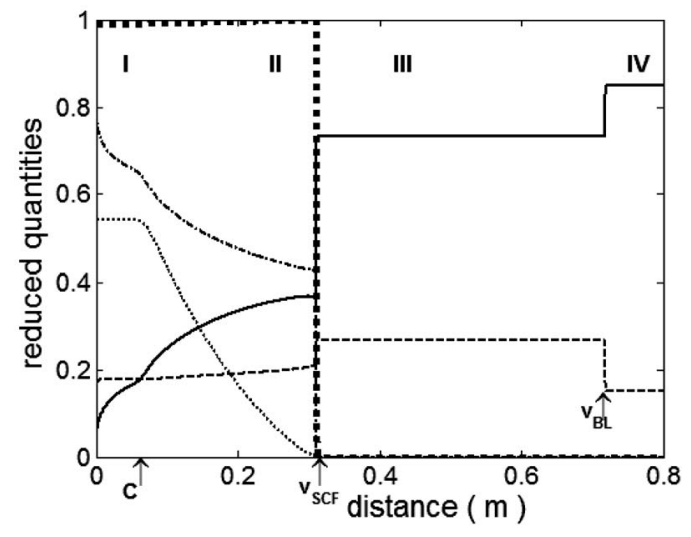

FIG. 8. Steam and dodecane displacement of dead oil. The water saturation, oil saturation, and steam saturation are shown as the dashed, solid, and dashed-dotted curves. The volatile-oil fraction in the oil phase and the reduced temperature are shown as the dotted and filled-square curves.

Buckley-Leverett shock with velocity $v_{B L}$ separates region II from region III.

Let us discuss the evolution of the volatile-oil bank. Initially there is no volatile-oil bank. It starts to be formed after injection. It grows as long as the volatile-oil-dead-oil mixture reaches the steam zone. The growth of the condensed volatile-oil bank stops as soon as no more volatile oil, carried by the liquid oil, can reach the steam zone.

Now we compare the case in Sec. VII C of heptane coinjection to the case in Sec. VII D where a mixture of dead oil and heptane is displaced by pure steam. For the coinjected $(20 \%)$ case a large oil bank is built up. On the other hand, with as much as $50 \%$ oil in the initial oil mixture the volatile-oil bank is very small. This is so because only the volatile oil that is stripped from the dead oil enters the steam zone and contributes to the building up of the volatile-oil bank for case (b). In particular, when the initial volatile-oil fraction is small it can take a long while before such a bank is built up, whereas such a building up is much faster with coinjected volatile oil.

\section{E. Steam and dodecane mixture displacing dead oil}

As shown in Fig. 8, besides the fast three-phase saturation rarefaction in region $\mathrm{I}$, there is a volatile-oil condensation wave in region II with velocity (20); in this wave both $T, u$ vary. At the state $C$ joining rarefactions in regions I and II the two characteristic speeds coincide. Region II is separated from region III by the SCF. Region III consists of a constant state, which is separated from region IV by a BuckleyLeverett shock.

Region I starts at the injection state $\left(S_{w c}, S_{g}=1\right.$ $\left.-S_{w c}, T^{i n j}, u^{i n j}\right)$ and it ends at the coincidence point $C$ $=\left(S_{w, C}, S_{g, C}, T^{i n j}, u^{i n j}\right)$, the left state of the condensation rarefaction. The three-phase rarefaction wave in region I is continued in region II as a condensation rarefaction wave, which is connected to the SCF. The left state $\left(S_{w, \mathrm{SCF}}^{-}, S_{g, \mathrm{SCF}}^{-}, T_{b}^{w}, u^{-}\right)$, the right state $\left(S_{w, \mathrm{SCF}}^{+}, S_{g}=0, T_{o}, u^{+}\right)$, and $v_{\mathrm{SCF}}$ satisfy the $\mathrm{RH}$ conditions (10)-(13). The SCF is left characteristic. 


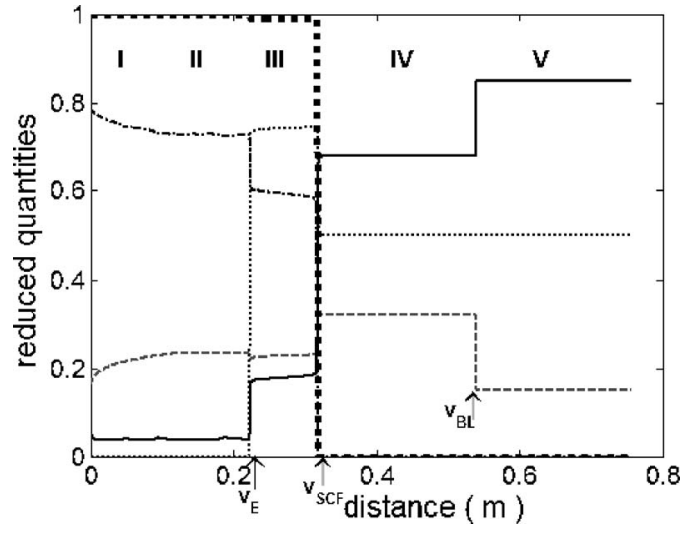

FIG. 9. Steam displacement of a dead-oil or dodecane mixture. The water saturation, oil saturation, and steam saturation are shown as the dashed, solid, and dashed-dotted curves. The volatile-oil fraction in the oil phase and the reduced temperature are shown as the dotted and filled-square curves.

\section{F. Pure steam displacing dead-oil and dodecane mixture}

As shown in Fig. 9, there are five regions. In region I, there is a three-phase saturation rarefaction. Near the injection point and at some other points very small transient effects are observed in the simulation. Region II consists of a constant state starting approximately at distance $0.1 \mathrm{~m}$. Separating region II from region III there is a dodecane evaporation shock, followed by a fast composition rarefaction wave. The evaporation shock speed $v_{E}$ coincides with the speed of the left part of the composition rarefaction wave. In region III the temperature and the total velocity are lower than in region I. Regions III and IV are separated by the SCF, which is left characteristic. The temperature drops to its initial reservoir value and the total velocity to its final downstream value. There is only a constant state in region IV. Regions IV and $\mathrm{V}$ are separated by a Buckley-Leverett shock with speed $v_{B L}$. Region $\mathrm{V}$ contains the initial saturations.

Region I starts at the injection state $\left(S_{w}, S_{g}, T, u\right)$ $=\left(S_{w c}, 1-S_{w c}, T_{b}^{w}, u^{i n j}\right)$ and finishes at $\left(S_{w, E}^{-}, S_{g, E}^{-}, T_{b}^{w}, u^{i n j}\right)$. This state also represents the left side of the dodecane evaporation shock as region II is a constant state. The dodecane evaporation shock has speed $v_{E}$ and the right state $E^{+}$ $=\left(S_{w, E}^{+}, S_{g, E}^{+}, T_{E}^{+}, u_{E}^{+}\right)$. Left and right states and $v_{E}$ satisfy the RH Eqs. (10)-(13). The evaporation shock is left characteristic too. Region III starts at $E^{+}$with a composition rarefaction, which ends at $\left(S_{w, \mathrm{SCF}}^{-}, S_{g, \mathrm{SCF}}^{-}, T_{E}^{-}, u_{E}^{-}\right)$, the left state of the SCF.

The right state of the $\mathrm{SCF}$ is $\mathrm{SCF}^{+}=\left(S_{w, \mathrm{SCF}}^{+}, S_{g, \mathrm{SCF}}^{+}\right.$ $\left.=0, T_{o}, u_{\mathrm{SCF}}^{+}\right)$. Left and right states and $v_{\mathrm{SCF}}$ satisfy the $\mathrm{RH}$ conditions. The SCF is left characteristic. Region IV consists of the constant state $\mathrm{SCF}^{+}$and ends with a Buckley-Leverett shock.

\section{G. Comparison of cases}

We can distinguish three important mass transfer waves, viz., the SCF, the evaporation wave upstream of the SCF, and the condensation wave downstream of the SCF. Inspection of the results reveals the crucial role of the speeds of these

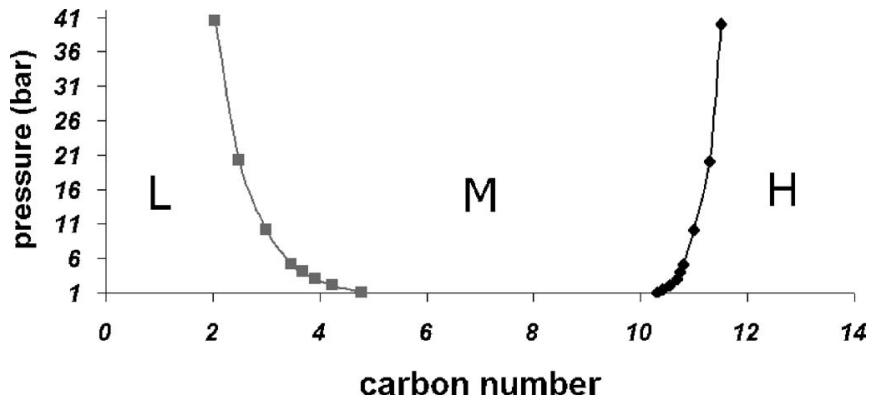

FIG. 10. Pressure vs carbon-number domain for low, medium, and high boiling-temperature alkanes. The structure of the solution changes at the curves separating the $L, M$, and $H$ regions.

waves, i.e., the speeds of the evaporation shock, the steam condensation shock, and the condensation wave (shock or rarefaction). For the medium boiling-point alkane (heptane) the three waves merge into a single wave, leading to high recovery. Both high recovery [5] and the existence of the volatile oil bank had been observed in the coinjection experiments [7]. Similar observations can be made for Willman's experiments [9], where medium boiling temperature oil was present initially. For the other cases the three waves spread out leading to lower recovery. These statements hold true irrespective of whether the volatile oil is present initially or coinjected.

For the high boiling-point alkane (dodecane) the condensation wave collapses on the SCF, whereas the evaporation wave separates from the SCF. This leads to some positive effect on the oil recovery.

For the low boiling-point alkane (cyclobutane) the evaporation wave collapses on the SCF, whereas the evaporation wave separates from the steam condensation shock. This has only a small effect on the oil recovery.

In summary, resonance, i.e., equality of wave speeds, leads to high amplitude waves, i.e., favorable recovery. This occurs for medium boiling-point alkanes.

We use these ideas to find the bifurcation loci in the pressure or carbon-number plane. We used an injected mass fraction (mass volatile-oil or total-mass) of $20 \%$. We derived polynomial expansions for the properties of volatile oil in terms of the carbon number, i.e., for the viscosity, the liquid heat capacity, the evaporation heat, the molar weight, the liquid density, and the boiling point. In this way we find not only the properties of the alkanes with integer carbon numbers but also of any pseudocompound, characterized by any real value for the carbon number. We carried out enough simulations to isolate the wave sequences typical of high, medium, or low boiling-temperature volatile oils. The resulting curves are shown in Fig. 10. The curves are accurate within a carbon-number change of 0.1 . The range of favorable medium temperature boiling point, where resonance occurs, becomes larger with increasing pressure.

In our simulation we injected $20 \mathrm{w} / \mathrm{w} \%$ volatile oil in the steam rather than $5 \%$ as we did in the laboratory experiments for reasons of clear illustration. For 5\% simulations the volatile-oil bank is thinner as expected, but the overall picture does not change (see Fig. 11). 

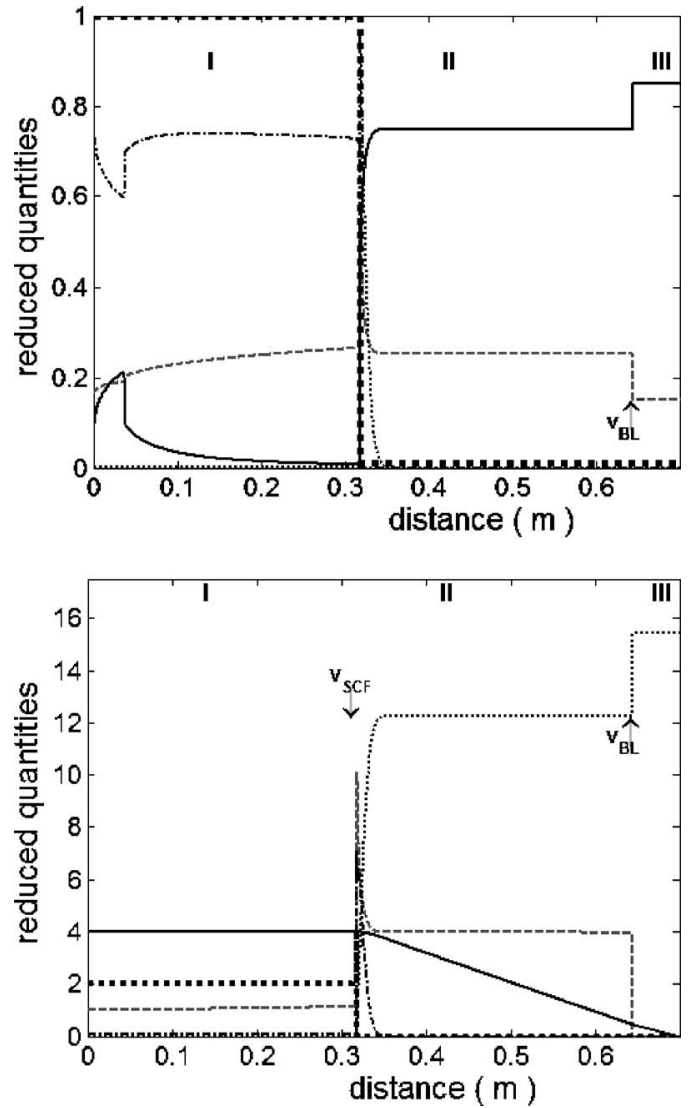

FIG. 11. Steam and heptane displacement of dead oil, using $5 \mathrm{w} / \mathrm{w} \%$. At the top, the water saturation, oil saturation, and steam saturation are shown as the dashed, solid, and dashed-dotted curves. The volatile oil fraction in the oil phase and the reduced temperature are shown as the dotted and filled-square curves. At the bottom, the mass fluxes for water, dead oil, and volatile oil are indicated as dashed, dotted, and dot-dashed curves, and the enthalpy flux and pressure as the filled squares and solid curves. The scaled pressure has small gradients in the vapor zone.

\section{CONCLUSIONS}

We developed a model that captures the main physical features of thermal three-phase flow, involving water, dead oil, and volatile oil. The numerical solution for different injected mixtures and initial oil composition reveals its structure in terms of rarefaction and shock waves. These waves are validated by verifying that they satisfy all properties predicted by mathematical analysis based on the mathematical theory of nonlinear conservation laws. In the solution found computationally numerical diffusion effects are controlled by using extremely small grid cells.

In the 1D setting, coinjection of medium boiling temperature volatile oil in steam leads to $100 \%$ recovery of oil (Fig. 6). This improvement is due to the formation of an increasingly long volatile-oil bank displacing the oil in place. The initial presence of medium boiling-temperature volatile oil also improves oil recovery (see Fig. 7). This is due to the formation of a thin volatile-oil bank displacing the oil in place. Clearly the volatile-oil bank displaces all the dead oil because they are in the same phase. This solution agrees with the observations found previously in the laboratory experiments. There is agreement between analytical, numerical, and experimental results.

As far as the recovery efficiency is concerned, the initial presence of medium boiling-temperature volatile oil has a positive effect. The initial presence of high boilingtemperature volatile oil has a much smaller effect (see Fig. 9). Coinjection of high boiling-temperature volatile oil in steam has a negligible effect (see Fig. 8). Coinjected or initially present low boiling-temperature volatile oil has no effect (see Figs. 4 and 5).

This model reveals that the essential mechanism for good recovery is that all the volatile oil condenses at the same point where the steam condenses. In mathematical language, this occurs when the evaporation and condensation shock speeds coincide with the speed of the steam condensation front. In physical language, high boiling-temperature volatile oil finds it difficult to evaporate and therefore the evaporation wave is slower than the steam condensation shock. The low temperature boiling-point volatile oil finds it difficult to condense and therefore the condensation wave is faster than the steam condensation shock. For the medium oil the evaporation and the condensation wave collapse on the steam condensation shock.

\section{ACKNOWLEDGMENTS}

This work follows the brilliant inspiration by Daan $\mathrm{N}$. Dietz. Experimental evidence was also gathered in the MSc. theses of A. Emke, G. Metselaer, J. W. Scholten, D. W. van Batenburg, R. Quak, and C. T. S. Palmgren. This paper was initiated at the Institute for Mathematics and its Applications in Minnesota with NSF support and completed with support from NWO-WOTRO (Stichting voor Wetenschappelijk Onderzoek van de Tropen). We thank C. J. van Duijn for his early participation in this effort. We are grateful to our librarian, Paul Suijker, for discovering details of the references. We thank the anonymous referees for valuable comments. We thank TU-Delft and IMPA for their hospitality and support.

\section{APPENDIX A: STEAM OR HEPTANE MIXTURE DISPLACING A DEAD OIL WITH LESS VOLATILE OIL}

To illustrate the effect of low $(5 \mathrm{w} / \mathrm{w} \%)$ concentration of volatile oil in the injected mixture we made the run shown at the bottom of Fig. 11. Clearly the wave structure is identical to that found in Fig. 6 . The difference is only that the dissolved volatile-oil peak is much thinner. Even for this lowinjection concentration the recovery is high, as shown by the small amount of oil left behind. However, we observe that the initialization effect, i.e., the transient bump of oil left behind near the injection point is larger because the volatileoil bank takes longer to build up.

The top part of Fig. 11 includes the fluxes for water, volatile oil, and dead oil, and also the pressure. We rescaled the values for clear illustration. However, all mass fluxes are on 
the same scale. Notice that the pressure gradients are much larger in the liquid zone.

\section{APPENDIX B: PHYSICAL QUANTITIES-SYMBOLS AND VALUES}

In this appendix we summarize the values and units of the various quantities used in the computation and empirical expressions for the various parameter functions. All enthalpies per unit mass are are taken relative to the reference temperature of the components in their standard form. All heat capacities are specified at constant pressure. All enthalpies in their standard form are zero at the reference temperature.

\section{Temperature-dependent properties of steam, water, and heptane}

We use Refs. [40-42] to obtain all the following properties. Properties for other volatile components, such as cyclobutane and dodecane can be derived from the same references. A conventional choice for the reference temperature is $\bar{T}=298.15 \mathrm{~K}$.

The rock enthalpy $H_{r}$ is

$$
(1-\varphi) C_{r}(T-\bar{T}), \quad C_{r}=3274000\left(\mathrm{~J} / \mathrm{m}^{3} \mathrm{~K}\right) .
$$
by

The liquid water enthalpy $h_{W}(T)[\mathrm{J} / \mathrm{kg}]$ is approximated

$$
h_{W}(T)=c_{W}(T-\bar{T}), \quad c_{W}=4184(\mathrm{~J} / \mathrm{kg} \mathrm{K}) .
$$

The heptane enthalpy $h_{o V}[\mathrm{~J} / \mathrm{kg}]$ and the dead-oil enthalpy $h_{o D}$ are approximated by

$$
h_{o V}(T)=c_{o V}(T-\bar{T}), \quad h_{o D}(T)=c_{o D}(T-\bar{T}) .
$$

The values for the heat capacities of heptane and dead oil are

$$
c_{o V}=2242, \quad c_{o D}=1914.1(\mathrm{~J} / \mathrm{kg} \mathrm{K}) .
$$

The liquid volatile-oil and dead-oil heat capacities are not the exact heat capacities, but slightly adapted so that the enthalpy of oil per unit volume is independent of composition. Therefore the heat capacity of the oleic phase per unit volume can also be defined independently of composition, leading to an oleic phase heat capacity per unit volume of

$$
C_{o}=1.531 \times 10^{6}\left(\mathrm{~J} / \mathrm{m}^{3} \mathrm{~K}\right) .
$$

The steam enthalpy $h_{g W}$ is given by

$$
h_{g W}(T)=h_{g W}^{s}(T)+\Lambda_{W}(\bar{T}),
$$

and the sensible steam enthalpy is approximated as

$$
h_{g W}^{s}(T)=c_{p g w}(T-\bar{T}), \quad c_{p g w}=1964(\mathrm{~J} / \mathrm{kg} \mathrm{K}) .
$$

The volatile-oil vapor enthalpy $h_{g V}$ as a function of temperature is given by

$$
h_{g V}(T)=h_{g V}^{s}(T)+\Lambda_{V}(\bar{T})
$$

and the sensible heptane enthalpy is approximated as

$$
h_{g V}^{s}(T)=c_{p g v}(T-\bar{T}), \quad c_{p g v}=1658(\mathrm{~J} / \mathrm{kg} \mathrm{K}) .
$$

For the latent heat $\Lambda_{W}(T)\left[\Lambda_{V}(T)\right](\mathrm{J} / \mathrm{kg})$ or evaporation heat of water (heptane) we use

$$
\Lambda_{W}=3105600-2220 T, \quad \Lambda_{V}=538830-584 T \text {. }
$$

The liquid-water viscosity $\mu_{w}[\mathrm{~Pa} \mathrm{~s}]$ is approximated by

$$
\mu_{w}=\exp (-12.06+1509 / T) \text {. }
$$

The viscosity of the dead oil $\mu_{o d}$ and heptane $\mu_{o v}$ are written as

$$
\mu_{\text {od }}=e^{-13.79+3781 / T}, \quad \mu_{o v}=e^{-10.813+880.2 / T},
$$

and the viscosity of the oleic phase is approximated by the quarter power rule

$$
\mu_{o}=\left(\frac{\rho_{o v}}{\rho_{V}} \mu_{o v}^{1 / 4}+\frac{\rho_{o d}}{\rho_{D}} \mu_{o d}^{1 / 4}\right)^{4} .
$$

We assume that the viscosity of the gas is independent of composition

$$
\mu_{g}=1.8264 \times 10^{-5}(T / 300)^{0.6} .
$$

The water saturation pressure is given by Eq. (3). The pure phase densities of steam and volatile-oil vapor are given by Eqs. (2) and the corresponding concentrations $\rho_{g w}, \rho_{g v}$ are given in Table. I

\section{Three-phase relative permeabilities}

We used Stone's expressions [31] for three-phase permeability: Eqs. (B14) and (B15) describe the water relative permeability $k_{r w}$, the gas-phase relative permeability $k_{r g}$, and the oil relative permeability $k_{r o}$, respectively. For convenience we have taken the residual oil parameter $S_{o m}$ used by Fayers [31] equal to zero. The relative permeabilities $k_{r w}, k_{r g}$ are functions solely of the water saturation $S_{w}$ and the gas saturation $S_{g}$, respectively.

$$
\begin{gathered}
k_{r w}=k_{r w}^{\prime} S_{w e}^{3+2 / \lambda}, \quad k_{r g}=k_{r g}^{\prime}\left(1-S_{g e}\right)^{2}\left(1-S_{g e}^{1+2 / \lambda}\right), \\
k_{r o}=\frac{S_{o}\left(1-S_{w c}\right)}{k_{r c o w}\left(1-S_{w}\right)\left(1-S_{w c}-S_{g}\right)} k_{r o w} k_{r o g}, \\
S_{w e}=\frac{S_{w}-S_{w c}}{1-S_{w c}-S_{o r}}, \quad S_{g e}=\frac{1-S_{g}-S_{w c}}{1-S_{w c}-S_{o r}} \\
k_{r o w}=k_{r g}^{\prime}\left(1-S_{w e}\right)^{2}\left(1-S_{w e}^{1+2 / \lambda}\right), \quad k_{r o g}=k_{r w}^{\prime} S_{g e}^{3+2 / \lambda} .
\end{gathered}
$$

We took $k_{r w}^{\prime}=1 / 2, k_{r g}^{\prime}=1$, and $k_{r c o w}=1$. Here $\lambda=0.5$ is the sorting factor, $S_{w c}$ given in the table and $S_{o r}=0$ are the connate water saturation and the residual oil saturation, respectively.

We can express the Buckley-Leverett fractional flow functions for $\alpha=o, w, g$ as 
TABLE I. Summary of physical input parameters and variables.

\begin{tabular}{|c|c|c|c|}
\hline Physical quantity & Symbol & Value & Unit \\
\hline Water, gas, oil fractional flows & $f_{w}, f_{g}, f_{o}$ & Eq. (B16). & $\mathrm{m}^{3} / \mathrm{m}^{3}$ \\
\hline Steam, volatile-oil enthalpy/unit mass & $h_{g W}, h_{g V}$ & Eqs. (B5) and (B7) & $\mathrm{J} / \mathrm{kg}$ \\
\hline Sensible enthalpy/unit mass & $h_{g W}^{s}, h_{g V}^{s}$ & Eqs. (B6) and (B8) & $\mathrm{J} / \mathrm{kg}$ \\
\hline Volatile-oil, oil enthalpy/unit mass & $h_{o V}, h_{o D}$ & Eqs. (B3) & $\mathrm{J} / \mathrm{kg}$ \\
\hline Gas enthalpy, oil enthaply & $H_{g}, H_{o}$ & $H_{g w}+H_{g v}, H_{o v}+H_{o d}$ & $\mathrm{~J} / \mathrm{m}^{3}$ \\
\hline Steam, volatile-oil enthalpy & $H_{g W}, H_{g V}$ & $\rho_{g W}(T) h_{g W}(T), \rho_{g V}(T) h_{g V}(T)$ & $\mathrm{J} / \mathrm{m}^{3}$ \\
\hline Sensible steam, volatile-oil enthalpy & $H_{g W}^{s}, H_{g V}^{s}$ & $\rho_{g W}(T) h_{g W}^{s}(T), \rho_{g V}(T) h_{g V}^{s}(T)$ & $\mathrm{J} / \mathrm{m}^{3}$ \\
\hline Partial steam, volatile-oil enthalpy & $H_{g w}, H_{g v}$ & $\rho_{g w}(T) h_{g W}(T), \rho_{g v}(T) h_{g V}(T)$ & $\mathrm{J} / \mathrm{m}^{3}$ \\
\hline Pure volatile-oil, dead-oil enthalpy & $H_{o V}, H_{o D}$ & $\rho_{V}(T) h_{o V}(T), \rho_{o D}(T) h_{o D}(T)$ & $\mathrm{J} / \mathrm{m}^{3}$ \\
\hline Volatile-oil, dead-oil enthalpy & $H_{o v}, H_{o d}$ & $\rho_{o v}(T) h_{o V}(T), \rho_{o d}(T) h_{o D}(T)$ & $\mathrm{J} / \mathrm{m}^{3}$ \\
\hline Rock enthalpy & $H_{r}$ & $C_{r}(T-\bar{T})$, Eq. $(\mathrm{B} 1)$ & $\mathrm{J} / \mathrm{m}^{3}$ \\
\hline Water enthalpy & $H_{W}$ & $\rho_{W}(T) h_{W}(T)$ & $\mathrm{J} / \mathrm{m}^{3}$ \\
\hline Porous rock permeability & $k$ & $1.0 \times 10^{-12}$ & $\mathrm{~m}^{2}$ \\
\hline Water, gas, oil relative permeabilities & $k_{r w}, k_{r g}, k_{r o}$ & Eqs. (B14) and (B15) & $\mathrm{m}^{3} / \mathrm{m}^{3}$ \\
\hline Molar weight, $\mathrm{H}_{2} \mathrm{O}, \mathrm{C}_{7} \mathrm{H}_{16}$, dead-oil & $M_{W}, M_{V}, M_{D}$ & $0.018,0.10021,0.4$ & $\mathrm{~kg} / \mathrm{mole}$ \\
\hline Total pressure & $P$ & $1.0135 \times 10^{5}$ & $\mathrm{~Pa}$ \\
\hline Atmospheric pressure & $P_{o}$ & $1.0135 \times 10^{5}$ & $\mathrm{~Pa}$ \\
\hline Partial pressures & $P_{w}, P_{v}$ & Eqs. (3) and (4) & $\mathrm{Pa}$ \\
\hline Water, vapor, oil saturations & $S_{w}, S_{g}, S_{o}$ & independent variables & $\mathrm{m}^{3} / \mathrm{m}^{3}$ \\
\hline Residual oil, connate water saturation & $S_{o r}, S_{w c}$ & $0,0.15$ & $\mathrm{~m}^{3} / \mathrm{m}^{3}$ \\
\hline Injection saturations & $S_{w}^{i n j}, S_{o}^{i n j}$ & input & $\mathrm{m}^{3} / \mathrm{m}^{3}$ \\
\hline Temperature & $T$ & independent variable & $\mathrm{K}$ \\
\hline Three-phase temperature & $T\left(x_{o v}=1\right)$ & Eq. (5) & $\mathrm{K}$ \\
\hline Reservoir, injection temperature & $T_{o}, T^{i n j}$ & $293,370-373$ & $\mathrm{~K}$ \\
\hline Boiling point of water, volatile oil & $T_{b}^{w}, T_{b}^{v}$ & $373.15,371.57$ for heptane & $\mathrm{K}$ \\
\hline Total Darcy velocity & $u$ & volume flux of all phases & $\mathrm{m}^{3} /\left(\mathrm{m}^{2} \mathrm{~s}\right)$ \\
\hline Total injection velocity & $u^{i n j}$ & injected volume flux & $\mathrm{m}^{3} /\left(\mathrm{m}^{2} \mathrm{~s}\right)$ \\
\hline Water, volatile-oil evaporation heat & $\Lambda_{W}, \Lambda_{V}$ & see Eqs. (B9) & $\mathrm{J} / \mathrm{kg}$ \\
\hline Water, steam, oil viscosity & $\mu_{w}, \mu_{g}, \mu_{o}$ & Eqs. (B10)-(B13) & $\mathrm{Pa} \mathrm{s}$ \\
\hline Water, steam, volatile-oil vapor density & $\rho_{W}, \rho_{g W}, \rho_{g V}$ & 998.2, Eqs. (2) & $\mathrm{kg} / \mathrm{m}^{3}$ \\
\hline Pure heptane, dead-oil densities & $\rho_{V}, \rho_{D}$ & 683,800 & $\mathrm{~kg} / \mathrm{m}^{3}$ \\
\hline Steam, volatile-oil vapor concentrations & $\rho_{g w}, \rho_{g v}$ & $\rho_{g W} P_{w} / P, \rho_{g V} P_{v} / P$, Eq. (2) & $\mathrm{kg} / \mathrm{m}^{3}$ \\
\hline Liquid volatile-oil concentrations & $\rho_{o v}, \rho_{o d}$ & obtained from Eqs. (6) and (1) & $\mathrm{kg} / \mathrm{m}^{3}$ \\
\hline Molar fraction volatile-oil in liquid-oil & $x_{o v}$ & Eqs. (5) and (4) & - \\
\hline Rock porosity & $\varphi$ & 0.38 & $\mathrm{~m}^{3} / \mathrm{m}^{3}$ \\
\hline
\end{tabular}

$$
f_{\alpha}=\left(k_{r \alpha} / \mu_{\alpha}\right) /\left(k_{r w} / \mu_{w}+k_{r o} / \mu_{o}+k_{r g} / \mu_{g}\right),
$$

where $f_{\alpha}$ is the fraction of the volume flux of phase $\alpha$ [26].

\section{APPENDIX C: ANALYSIS OF THE CHARACTERISTIC EQUATIONS}

We observe that making the determinant of Eq. (17) equal to zero leads to a polynomial equation of third order in $\lambda$ $=\eta / u$ and thus we get (if all solutions are real) a slow, a medium, and a fast wave solution.

We want to eliminate the first two elements of the fourth row of Eq. (17). To do so we find $x$ and $y$ such that $x F_{11}$ $+y F_{31}+F_{41}=0$ with $F_{i j}$ given in Eq. (D4) and we obtain

$$
y=\frac{H_{g}-H_{o}+\frac{\rho_{g w}}{\rho_{W}}\left(H_{o}-H_{w}\right)}{1-\frac{\rho_{g v}}{\rho_{V}}-\frac{\rho_{g w}}{\rho_{W}}},
$$

$$
x=\frac{H_{g}-H_{w}-\frac{\rho_{g v}}{\rho_{V}}\left(H_{o}-H_{w}\right)}{1-\frac{\rho_{g v}}{\rho_{V}}-\frac{\rho_{g w}}{\rho_{W}}} .
$$

Remembering that $G_{i j}$ are given in Eqs. (D3), it is easy to verify for $\alpha=1,2$, 


$$
x F_{12}+y F_{32}+F_{42}=0, \quad x G_{1 \alpha}+y G_{3 \alpha}+G_{4 \alpha}=0 .
$$

Now $\mathcal{F}_{43}=x F_{13}+y F_{33}+F_{43}$ in the system (17) becomes

$$
\mathcal{F}_{43}=\Delta H_{T} f_{g}+H_{o}^{\prime}+\left(H_{w}^{\prime}-H_{o}^{\prime}\right) f_{w}+\left(H_{g}^{\prime}-H_{o}^{\prime}\right) f_{g},
$$

where

$$
\Delta H_{T}=x\left(\frac{\rho_{g w}}{\rho_{W}}\right)^{\prime}+y\left(\frac{\rho_{g v}}{\rho_{V}}\right)^{\prime} .
$$

The primes indicate differentiation relative to temperature. Analogously, $\mathcal{G}_{43}=x G_{13}+y G_{33}+G_{43}$ becomes

$$
H_{r}^{\prime}+\varphi\left\{\Delta H_{T} S_{g}+\left[H_{o}^{\prime}+\left(H_{w}^{\prime}-H_{o}^{\prime}\right) S_{w}+\left(H_{g}^{\prime}-H_{o}^{\prime}\right) S_{g}\right]\right\} .
$$

Finally $\mathcal{F}_{4}=x F_{1}+y F_{3}+F_{4}$ becomes

$$
\mathcal{F}_{4}=\frac{H_{g}-\frac{\rho_{g w}}{\rho_{W}} H_{w}-\frac{\rho_{g v}}{\rho_{V}} H_{o}}{1-\frac{\rho_{g v}}{\rho_{V}}-\frac{\rho_{g w}}{\rho_{W}}} .
$$

Now we are ready for the Gaussian elimination. In Eq. (17) we add to the fourth row the first row multiplied by $x$ and the third row multiplied by $y$ leading to

$$
\left(\begin{array}{cccc}
F_{11}-\lambda G_{11} & F_{12}-\lambda G_{12} & F_{13}-\lambda G_{13} & F_{1} \\
F_{21}-\lambda G_{21} & F_{22}-\lambda G_{22} & F_{23}-\lambda G_{23} & F_{2} \\
F_{31}-\lambda G_{31} & F_{32}-\lambda G_{32} & F_{33}-\lambda G_{33} & F_{3} \\
0 & 0 & \mathcal{F}_{43}-\lambda \mathcal{G}_{43} & \mathcal{F}_{4}
\end{array}\right)
$$

The rest of the Gaussian elimination is just as tedious and straightforward. The result is the matrix on the left-hand side of Eq. (18), where

$$
\begin{gathered}
A_{13}(\lambda) \equiv f_{13}^{\prime}+\left(\left(\frac{\rho_{g w}}{\rho_{W}}\right)^{\prime}-\frac{\rho_{g w}}{\rho_{W}} \frac{\left(\frac{\rho_{g v}}{\rho_{V}}\right)^{\prime}+\left(\frac{\rho_{g w}}{\rho_{W}}\right)^{\prime}}{\frac{\rho_{g v}}{\rho_{V}}+\frac{\rho_{g w}}{\rho_{W}}-1}\right)\left(f_{g}-\lambda \varphi S_{g}\right), \\
A_{33}(\lambda)=\left(\begin{array}{c}
\frac{\left(\frac{\rho_{g v}}{\rho_{V}}\right)^{\prime}+\left(\frac{\rho_{g w}}{\rho_{W}}\right)^{\prime}}{\rho_{V}}+\frac{\rho_{g w}}{\rho_{W}}-1 \\
\rho^{\prime}
\end{array}\right)\left(f_{g}-\lambda \varphi S_{g}\right)+f_{g}^{\prime}, \\
A_{3}=\frac{1}{\frac{\rho_{g v}}{\rho_{V}}+\frac{\rho_{g w}}{\rho_{W}}-1}+f_{g}, \\
A_{1}=f_{w}+\frac{\rho_{g w}}{1-\frac{\rho_{g v}}{\rho_{V}}-\frac{\rho_{g w}}{\rho_{W}}} .
\end{gathered}
$$

Using $v_{o d}=1-v_{o v}$ we write

$$
A_{23}^{0}=v_{o d} f_{g} \frac{\left(\frac{\rho_{g v}}{\rho_{V}}\right)^{\prime}\left(1-\frac{\rho_{g w}}{\rho_{W}}\right)+\left(\frac{\rho_{g w}}{\rho_{W}}\right)^{\prime} \frac{\rho_{g v}}{\rho_{V}}}{1-\frac{\rho_{g v}}{\rho_{V}}-\frac{\rho_{g w}}{\rho_{W}}}-v_{o v}^{\prime} f_{o},
$$

$$
A_{23}^{1}=v_{o d} \varphi S_{g} \frac{\left(\frac{\rho_{g v}}{\rho_{V}}\right)^{\prime}\left(1-\frac{\rho_{g w}}{\rho_{W}}\right)+\left(\frac{\rho_{g w}}{\rho_{W}}\right)^{\prime} \frac{\rho_{g v}}{\rho_{V}}}{1-\frac{\rho_{g v}}{\rho_{V}}-\frac{\rho_{g w}}{\rho_{W}}}+\varphi v_{o v}^{\prime} S_{o},
$$

$$
A_{2}=F_{2}+v_{o v} A_{1}-A_{3}\left(\frac{\rho_{g v}}{\rho_{V}}-v_{o, v}\right) .
$$

These results are used in Sec. IV B 1

\section{APPENDIX D: FLUX, ACCUMULATION FUNCTIONS, AND DERIVATIVES}

We use Eqs. (8) and (7). We chose to divide the first and second of Eqs. (8) by $\rho_{W}$ and $\rho_{V}$, respectively. Thus we obtain Eq. (9). The accumulation functions $G_{i}$ take the following form for water, volatile oil, total oil, and energy, respectively,

$$
\begin{gathered}
G_{w}=\varphi\left(S_{w}+\frac{\rho_{g w}}{\rho_{W}}(T) S_{g}\right), \\
G_{v}=\varphi v_{o v}(T)\left(1-S_{w}-S_{g}\right)+\frac{\rho_{g v}}{\rho_{V}}(T) S_{g}, \\
G_{o}=\varphi\left[1-S_{w}+\left(\frac{\rho_{g v}}{\rho_{V}}(T)-1\right) S_{g}\right], \\
G_{T}=H_{r}+\varphi\left[H_{o}+\left(H_{w}-H_{o}\right) S_{w}+\left(H_{g}-H_{o}\right) S_{g}\right],
\end{gathered}
$$

where all enthalpies are functions of the temperature. Similarly, the flow functions $F_{i}$ take the form

$$
\begin{gathered}
F_{w}=f_{w}+\frac{\rho_{g w}}{\rho_{W}}(T) f_{g}, \\
F_{v}=v_{o v}(T)\left(1-f_{w}-f_{g}\right)+\frac{\rho_{g v}}{\rho_{V}}(T) f_{g}, \\
F_{o}=\left(1-f_{w}-f_{g}\right)+\frac{\rho_{g v}}{\rho_{V}} f_{g}, \\
F_{T}=H_{o}+\left(H_{w}-H_{o}\right) f_{w}+\left(H_{g}-H_{o}\right) f_{g} .
\end{gathered}
$$

The partial derivatives of the accumulations (D1) are

$$
G_{11}=\varphi, \quad G_{12}=\varphi \frac{\rho_{g w}}{\rho_{W}}, \quad G_{13}=\varphi\left(\frac{\rho_{g w}}{\rho_{W}}\right)^{\prime} S_{g},
$$




$$
\begin{gathered}
G_{21}=-\varphi v_{o v}, \quad G_{22}=\varphi\left(\frac{\rho_{g v}}{\rho_{V}}-v_{o v}\right), \\
G_{23}=\varphi v_{o v}^{\prime}\left(1-S_{w}\right)+\varphi\left[\left(\frac{\rho_{g v}}{\rho_{V}}\right)^{\prime}-v_{o, v}^{\prime}\right] S_{g}, \\
G_{31}=-\varphi, \quad G_{32}=\varphi\left(\frac{\rho_{g v}}{\rho_{V}}-1\right), \quad G_{33}=\varphi\left(\frac{\rho_{g v}}{\rho_{V}}\right)^{\prime} S_{g}, \\
G_{41}=\varphi\left(H_{w}-H_{o}\right), \quad G_{42}=\varphi\left(H_{g}-H_{o}\right), \\
G_{43}=H_{r}^{\prime}+\varphi\left[H_{o}^{\prime}+\left(H_{w}^{\prime}-H_{o}^{\prime}\right) S_{w}+\left(H_{g}^{\prime}-H_{o}^{\prime}\right) S_{g}\right], \quad(\mathrm{D} 3) \\
\text { and the partial derivatives of the flow functions }(\mathrm{D} 2) \text { are } \\
F_{11}=\frac{\partial f_{w}}{\partial S_{w}}+\frac{\rho_{g w}}{\rho_{W}} \frac{\partial f_{g}}{\partial S_{w}}, \quad F_{12}=\frac{\partial f_{w}}{\partial S_{g}}+\frac{\rho_{g w}}{\rho_{W}} \frac{\partial f_{g}}{\partial S_{g}}, \\
F_{13}=f_{w}^{\prime}+\left(\frac{\rho_{g w}}{\rho_{W}}\right)^{\prime} f_{g}+\frac{\rho_{g w}}{\rho_{W}} f_{g}^{\prime}, \\
F_{21}=-v_{o v} \frac{\partial f_{w}}{\partial S_{w}}+\left(\frac{\rho_{g v}}{\rho_{V}}-v_{o v}\right) \frac{\partial f_{g}}{\partial S_{w}}, \\
F_{22}=-v_{o v} \frac{\partial f_{w}}{\partial S_{g}}+\left(\frac{\rho_{g v}}{\rho_{V}}-v_{o v}\right) \frac{\partial f_{g}}{\partial S_{g}},
\end{gathered}
$$

$$
\begin{gathered}
F_{23}=v_{o v}^{\prime} f_{o}+\left(\frac{\rho_{g v}}{\rho_{V}}\right)^{\prime} f_{g}-v_{o v} f_{w}^{\prime}+\left(\frac{\rho_{g v}}{\rho_{V}}-v_{o v}\right) f_{g}^{\prime}, \\
F_{31}=-\left(\frac{\partial f_{w}}{\partial S_{w}}+\frac{\partial f_{g}}{\partial S_{w}}\right)+\frac{\rho_{g v}}{\rho_{V}} \frac{\partial f_{g}}{\partial S_{w}}, \\
F_{32}=-\left(\frac{\partial f_{w}}{\partial S_{g}}+\frac{\partial f_{g}}{\partial S_{g}}\right)+\frac{\rho_{g v}}{\rho_{V}} \frac{\partial f_{g}}{\partial S_{g}}, \\
F_{33}=\left(\frac{\rho_{g v}}{\rho_{V}}\right)^{\prime} f_{g}+\frac{\rho_{g v}}{\rho_{V}} f_{g}^{\prime}-f_{w}^{\prime}-f_{g}^{\prime},
\end{gathered}
$$

$$
\begin{gathered}
F_{41}=\left(H_{w}-H_{o}\right) \frac{\partial f_{w}}{\partial S_{w}}+\left(H_{g}-H_{o}\right) \frac{\partial f_{g}}{\partial S_{w}}, \\
F_{42}=\left(H_{w}-H_{o}\right) \frac{\partial f_{w}}{\partial S_{g}}+\left(H_{g}-H_{o}\right) \frac{\partial f_{g}}{\partial S_{g}}, \\
F_{43}=H_{o}^{\prime}+\left(H_{w}^{\prime}-H_{o}^{\prime}\right) f_{w}+\left(H_{g}^{\prime}-H_{o}^{\prime}\right) f_{g} \\
+\left(H_{w}-H_{o}\right) f_{w}^{\prime}+\left(H_{g}-H_{o}\right) f_{g}^{\prime} .
\end{gathered}
$$

We use the convention that primes mean the derivative relative to temperature; the subscripts $w, v, o, T$ were replaced by $1,2,3,4$. These results are used in Appendix C.
[1] E. J. Hanzlik and D. S. Mims, in Proceedings of SPE International Improved Oil Recovery Conference in Asia, Kuala Lumpur, Malaysia (Publ. Society of Petroleum Engineers, Richardson, TX, 2003), SPE 84848.

[2] C. Betz, A. Farbar, C. M. Green, H. P. Koschitzky, and R. Schmidt, in Proceedings of ConSoil'98 (Thomas Telford, London, 1998), pp. 575-584.

[3] S. F. Kaslusky and K. S. Udell, J. Contam. Hydrol. 55, 213 (2002).

[4] K. S. Udell and L. D. Stewart, Conference of the International Association of Hydrogeologists, Calgary, Canada, 1990.

[5] J. Bruining, D. N. Dietz, A. Emke, G. Metselaar, and J. W. Scholten, in Proceedings I of the Third European Meeting on Improved Oil Recovery, Rome (Publ. Agip S.p.A., Rome, 1985), pp. 371-378.

[6] S. M. Farouq Ali and B. Abad, J. Cdn. Pet. Tech. 15(3), 80 (1976).

[7] J. Bruining, D. W. van Batenburg, H. J. de Haan, R. Quak, and C. T. S. Palmgren, in Proceedings of the Fourth Symposium on Enhanced Oil Recovery (Deuthsche Wissenschaftliche Gesellschaft für Erdöl, Ergas und Kohle, Hamburg, 1987), pp. 887-898.

[8] W. R. Shu and K. J. Hartman, SPE Reservoir Eng. 3(2), 457 (1988)

[9] B. T. Willman, V. V. Valeroy, G. W. Runberg, and A. J. Cornelius, J. Pet. Technol. 13, 681 (1961).
[10] J. Bruining and D. Marchesin, Transp. Porous Media 62, 251 (2006).

[11] J. Bruining, D. Marchesin, and S. Schechter, Qualitative Theory of Dynamical Systems 5, 81 (2004).

[12] J. Bruining, D. Marchesin, and C. J. van Duijn, Comput. Appl. Math. 22, 359 (2004); Informes de Matemática (IMPAPreprints), www.preprint.impa.br

[13] J. Bruining and C. J. van Duijn, Comput. Geosci. 4, 65 (2000).

[14] G. Mandl and C. W. Volek, SPEJ 9(1), 59 (1969).

[15] N. D. Shutler, SPEJ 12(6), 489 (1972).

[16] J. S. Wingard and F. M. Orr, SPE Advanced Technology Series 2, 167 (1994).

[17] Y. C. Yortsos, SPEJ 24(4), 458 (1984).

[18] R. Juanes and T. W. Patzek, Transp. Porous Media 55, 47 (2004).

[19] C. M. Dafermos, Hyperbolic Conservation Laws in Continuum Physics (Springer-Verlag, Berlin, 2000).

[20] J. Smoller, Shock Waves and Reaction-Diffusion Equations (Springer-Verlag, New York, 1980).

[21] A. H. Falls and W. M. Schulte, SPE Reservoir Eng. 7, 377 (1992).

[22] R. E. Guzman and F. J. Fayers, SPEJ 2, 301 (1997).

[23] E. Isaacson, D. Marchesin, and B. Plohr, SIAM J. Math. Anal. 21, 837 (1990).

[24] E. Isaacson, D. Marchesin, B. Plohr, and J. B. Temple, Mat. Apl. Comput. 2, 147 (1992). 
[25] D. Marchesin and B. J. Plohr, SPEJ 6, 209 (2001), SPE 71314.

[26] J. Bear, Dynamics of Fluids in Porous Media (Dover Publications, Inc., Dover, 1972).

[27] J. Bruining and C. J. van Duijn, Comput. Geosci. 10, 373 (2006).

[28] K. H. Coats, SPEJ 18(5), 369 (1978).

[29] V. Oballa, D. A. Coombe, and W. L. Buchanan, SPE Reservoir Eng. 5, 549 (1990).

[30] R. H. Brooks and A. T. Corey, J. Irrig. and Drain. Div. 6, 61 (1966).

[31] F. J. Fayers and J. D. Matthews, SPEJ 24(2), 224 (1984).

[32] W. J. Moore, Physical Chemistry (Prentice Hall, Englewood Cliffs, NJ, 1962).

[33] R. B. Bird, W. E. Stewart, and E. N. Lightfoot, Transport Phenomena (John Wiley, New York, 1960).
[34] L. W. Lake, Enhanced Oil Recovery (Prentice Hall International, Englewood Cliffs, NJ, 1989).

[35] G. A. Pope, SPEJ 20(3), 191 (1980).

[36] F. G. Hirasaki, SPEJ 21(2), 191 (1981).

[37] R. E. Dumoré, J. Hagoort, and A. S. Risseeuw, SPEJ 24(2), 169 (1984).

[38] S. L. Mitchell, K. W. Morton, and A. Spence, SIAM J. Sci. Comput. (USA) 27, 1202 (2006).

[39] B. J. Plohr (private communication).

[40] W. S. Tortike and S. M. Farouq-Ali, SPE Reservoir Eng. 4(4), 471 (1989).

[41] C. L. Yaws, Chemical Properties Handbook (McGraw-Hill, New York, 1999).

[42] R. C. Weast, CRC Handbook of Chemistry and Physics, 58th ed. (CRC Press, Inc., New York, 1977-1978). 